\title{
Inflammation-responsive delivery systems for the treatment of chronic inflammatory diseases
}

\author{
Zhengyu Deng ${ }^{1} \mathbb{D} \cdot$ Shiyong Liu ${ }^{1}(\mathbb{C}$
}

Accepted: 3 April 2021 / Published online: 15 April 2021

(c) Controlled Release Society 2021

\begin{abstract}
Inflammation is the biological response of immune system to protect living organisms from injurious factors. However, excessive and uncontrolled inflammation is implicated in a variety of devastating chronic diseases including atherosclerosis, inflammatory bowel disease (IBD), and rheumatoid arthritis (RA). Improved understanding of inflammatory response has unveiled a rich assortment of anti-inflammatory therapeutics for the treatment and management of relevant chronic diseases. Notwithstanding these successes, clinical outcomes are variable among patients and serious adverse effects are often observed. Moreover, there exist some limitations for clinical anti-inflammatory therapeutics such as aqueous insolubility, low bioavailability, off-target effects, and poor accessibility to subcellular compartments. To address these challenges, the rational design of inflammation-specific drug delivery systems (DDSs) holds significant promise. Moreover, as compared to normal tissues, inflamed tissue-associated pathological milieu (e.g., oxidative stress, acidic pH, and overexpressed enzymes) provides vital biochemical stimuli for triggered delivery of anti-inflammatory agents in a spatiotemporally controlled manner. In this review, we summarize recent advances in the development of anti-inflammatory DDSs with built-in pathological inflammation-specific responsiveness for the treatment of chronic inflammatory diseases.
\end{abstract}

Keywords Inflammation · Inflammatory microenvironment · Drug delivery systems (DDSs) · Stimuli-responsive polymers $\cdot$ Anti-inflammatory agents $\cdot$ Oxidative stress

\section{Introduction}

Inflammation is a dynamic immune process mounted by the innate and adaptive immune system to protect living organisms from injurious factors, including viruses (e.g., SARSCoV-2), bacteria, fungi, or dead cells [1-3]. In its controlled form (i.e., acute inflammation), it serves to eliminate pathogens, cellular debris, and inflammatory mediators from organisms, and stimulate tissue repair, resulting in resolution of inflammation and restoration of tissue homeostasis [1]. However, when allowed to proceed unchecked, inflammation may lead to autoimmune or autoinflammatory disorders, such as atherosclerosis, type 2 diabetes, inflammatory bowel

Shiyong Liu

sliu@ustc.edu.cn

1 CAS Key Laboratory of Soft Matter Chemistry, Hefei National Laboratory for Physical Sciences At the Microscale, Department of Polymer Science and Engineering, University of Science and Technology of China, 96 Jinzhai Road, Hefei 230026, Anhui Province, China disease (IBD), cystic fibrosis (CF), rheumatoid arthritis (RA), Alzheimer's disease (AD), and so forth [1-3]. In such chronic inflammatory diseases, the exact identification of the inflammatory stimulus is often unrevealed and, if revealed, is demanding to eradicate [1-3]. Therefore, it is of great significance to suppress excessive inflammation and enhance resolution via therapeutically targeting chronic inflammatory responses [4].

Increasing understanding of inflammatory response has unveiled a rich assortment of anti-inflammatory agents, spanning from aspirin, nonsteroidal anti-inflammatory drugs (NSAIDs), glucocorticoids, anti-cytokine biologicals to therapeutic nucleic acids for the treatment and management of chronic diseases [5]. Notwithstanding these successes, clinical outcomes are variable especially when inflammatory diseases have been established and serious adverse effects are common as a result of compromised host defense. For instance, the risks of additional infections increase for those patients taking antibodies against tumor necrosis factor- $\alpha$ (TNF- $\alpha$ ) as anti-inflammatory therapy [1]. Moreover, there exist some limitations such as aqueous insolubility (e.g., 
NSAIDs and glucocorticoids), low bioavailability, off-target effects, and poor accessibility to subcellular compartments [6-8]. To resolve these issues of anti-inflammatory agents, the rational development of inflammation-specific and inflammation-responsive drug delivery systems (DDSs) has been proposed [6-8].

Clinical inflammation is well known and recognized by classical signs of heat, redness, swelling, pain, and loss of function, and these "macroscopic" features are governed by complex immune-regulatory pathways [7]. For example, the recognition of danger signals by host cells triggers the release of proinflammatory cytokines and chemokines, resulting in increased vascular permeability and angiogenesis (heat, redness, and swelling) [7], which is reminiscent of well-known enhanced permeability and retention (EPR) effect observed in solid tumors [9, 10]. In fact, extravasation through leaky vasculature and ensuing inflammatory cell-mediated sequestration (ELVIS) effect [7, 11, 12] for macromolecules or nano-objects in inflamed tissues was firstly corroborated in patients with RA [13]. Nevertheless, there is overwhelming evidence that numerous cell surface receptors, such as cell adhesion molecules (CAMs), integrins, folate receptor- $\beta$ (FR- $\beta$ ), and mannose receptor, are upregulated within inflamed areas upon inflammatory stimulation [7]. Taken together, these ubiquitous hallmarks render it possible the design of inflamed tissue-selective antiinflammatory therapy by leveraging passive and/or active targeting approaches [7, 8]. On the other hand, as compared to healthy tissues, pathologically inflamed tissue-associated biochemical signals comprise high oxidative stress (e.g., reactive oxygen species, ROS, and reactive nitrogen species, RNS), acidic $\mathrm{pH}(\sim 6.4)$, and overexpressed enzymes (e.g., cyclooxygenases (COXs) and matrix metalloproteinase (MMPs)), enabling the development of inflammationtriggered drug delivery platforms $[14,15]$.

So far, quite a few reviews have been presented to summarize inflammation-specific nanotherapeutics, bioinspired nanocarriers, and oxidation-responsive nanomaterials for the treatment of a variety of chronic inflammatory diseases [16-23]. However, systematic overviews of antiinflammatory DDSs with built-in responsiveness to pathological inflammation-related biochemical signals remain limited [14, 15, 24]. In this contribution, we summarize recent progress on inflammation-responsive DDSs as promising anti-inflammatory therapy for chronic inflammatory diseases. The main body of this review is organized according to the responsive properties of systems to specific types of inflammation-associated stimuli, comprising of oxidationresponsive, $\mathrm{pH}$-responsive, enzyme-responsive, and multistimuli-responsive systems (Scheme 1). Besides, several cytoplasmic reductive milieu-responsive anti-inflammatory DDSs are also covered (Scheme 1). It is worth mentioning that we provide a summary of the most relevant and pioneering reports by highlighting selected and representative examples throughout the main text. Finally, a brief conclusion section is presented to outline the future challenges in this promising field.

\section{Inflammation-responsive DDSs}

\section{Oxidation-responsive and oxidative stress-eliminating systems}

As mentioned above, oxidative stress, as a result of the imbalance between oxidants (e.g., ROS and RNS) and antioxidants (e.g., glutathione (GSH) and vitamin E), is involved in both acute and chronic inflammatory responses [21, 25, 26]. Specifically, highly reactive ROS including superoxide anion $\left(\mathrm{O}_{2}{ }^{--}\right)$, hydrogen peroxide $\left(\mathrm{H}_{2} \mathrm{O}_{2}\right)$, hydroxy radical $\left(\mathrm{HO}^{\circ}\right)$, and hypochlorous acid $(\mathrm{HOCl})$, are closely connected to RNS, such as nitric oxide (NO) and peroxynitrite $\left(\mathrm{ONOO}^{-}\right)[21,26]$. Concerning higher equilibrium levels of ROS and RNS, a growing body of literature has been reported to sense, leverage, and modulate oxidative stress for the imaging and treatment of chronic inflammatory diseases $[15,23,25,27]$. In this part, we will present an overview of oxidation-responsive and oxidative stress-eliminating systems according to the types of reactive moieties.

\section{Boronate-containing systems}

Boronic ester motif is well known to be degraded by oxidation with elevated levels of ROS or RNS at inflammation and tumor sites, and this triggering chemistry has been extensively exploited for the development of oxidation-sensitive nanocarriers for biomedical applications [28-32]. In this respect, Zhang and coworkers systematically explored the therapeutic potentials of nanoplatforms consisting of phenylboronic ester-conjugated $\beta$-cyclodextrin $(\beta$-CD) for the management of chronic inflammatory diseases [33-38]. For instance, oxidation-responsive nanoparticles encapsulating antioxidant TEMPOL (a typical free radical scavenger) were fabricated using phenylboronic ester-modified $\beta$-CD, lecithin, and 1,2-distearoyl-sn-glycero-3-phosphoethanolamine- $N$-poly (ethylene glycol) (DSPE-PEG; number-average molecular weight (MW) of PEG segment is $2000 \mathrm{Da}$ ) through nanoprecipitation method, collectively serving as superoxide dismutase (SOD)/catalase (CAT) mimics [34, 39]. Accordingly, hydrophobic and $\mathrm{H}_{2} \mathrm{O}_{2}$-reactive $\beta$-CD derivatives within drug-loaded nanovehicles could transform into water-soluble products upon contact with oxidative environment, leading to consumption of ROS (e.g., $\mathrm{H}_{2} \mathrm{O}_{2}$ ), dissociation of nanovehicles, and release of TEMPOL. Significantly, it was revealed that such SOD/CAT mimetic nanosystem was efficiently accumulated in the 
Scheme 1 Inflammationspecific delivery systems capable of responding to pathological stimuli such as oxidative stress, $\mathrm{pH}$ conditions, overexpressed enzymes, and intracellular reductive milieu towards the treatment and management of diverse chronic diseases

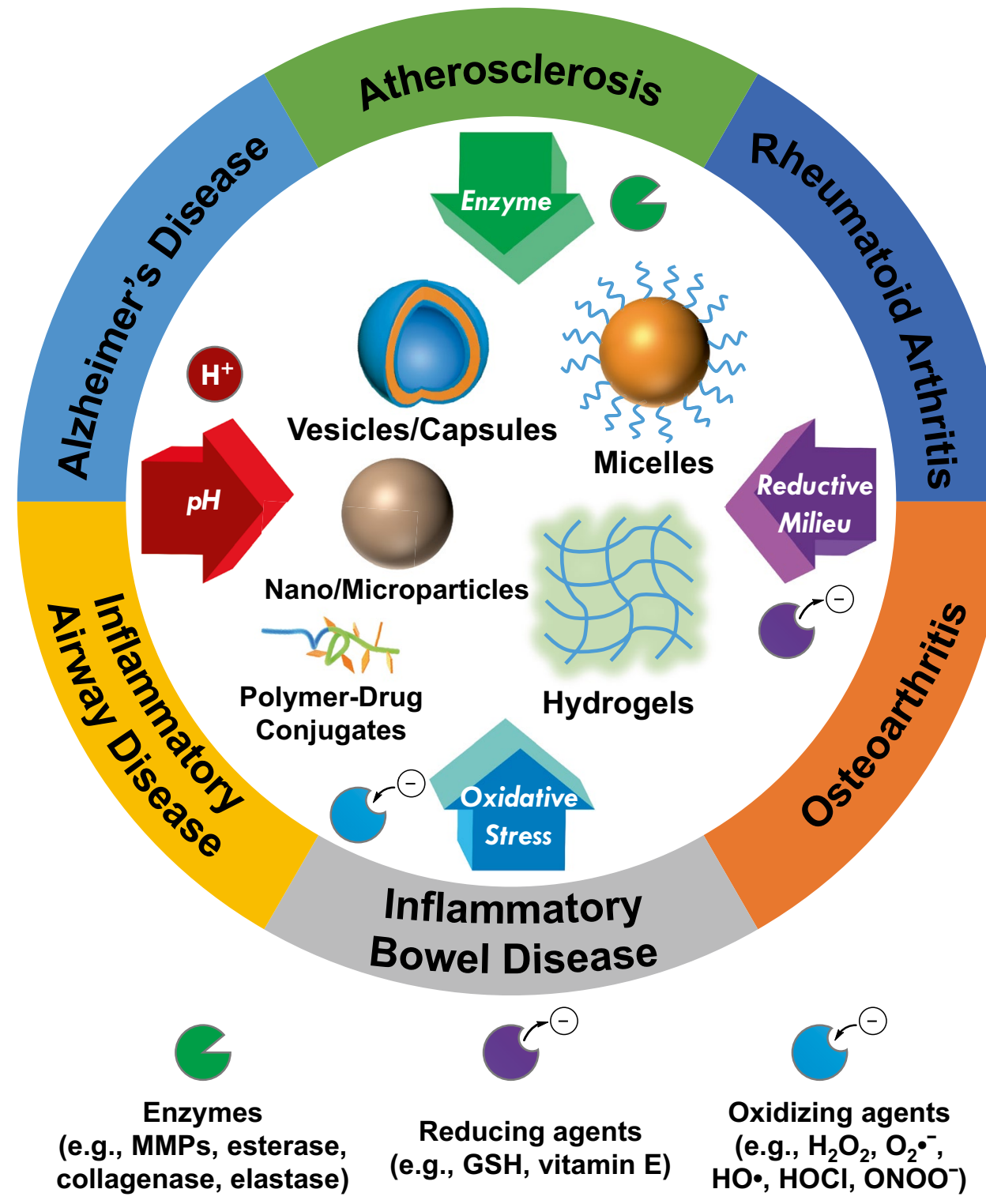

inflamed colon in vivo with reduced nonselective distribution in healthy organs after oral administration [40]. In three mice colitis models, these oxidative stress-eliminating nanomedicine notably mitigated manifestations and suppressed expressions of proinflammatory mediators, with superior therapeutic efficacy over free TEMPOL and poly(lactide-coglycolide) (PLGA)-based nonresponsive nanosystem.

In their follow-up work, TEMPOL and phenylboronic ester were covalently attached onto $\beta$-CD, followed by a coassembly process in combination with lecithin and DSPE-PEG, affording broad-spectrum ROS-scavenging nanoparticles (Fig. 1) [37, 38]. By eliminating multiple ROS, including $\mathrm{H}_{2} \mathrm{O}_{2}, \mathrm{O}_{2}{ }^{--}, \mathrm{HO}^{\bullet}$, and $\mathrm{HOCl}$ (Fig. 1b), these combinatorial nanoparticles could effectively attenuate oxidative stress-induced inflammation and cell apoptosis as well as inhibit oxidized low-density lipoprotein
(oxLDL)-induced foam cell formation, which was closely associated with the pathogenesis of atherosclerosis. It was demonstrated that such anti-inflammatory nanoparticles accumulated in atherosclerotic lesions in apolipoprotein E-deficient $\left(\mathrm{ApoE}^{-I^{-}}\right)$mice through the ELVIS effect and significantly inhibited the development of atherosclerosis following intravenous administration [37]. In another separate study, ROS-responsive and hydrophobic phenylboronic ester-conjugated $\alpha$-cyclodextrin $(\alpha$-CD) derivatives were synthesized and then used to physically encapsulate anti-rheumatic drug dexamethasone (Dex), in combination with folic acid-modified DSPE-PEG (DSPEPEG-FA) to fabricate FA-decorated nanoparticles for the treatment of RA [41]. Preliminary in vivo evaluations suggested that such targeted nanoparticles were effectively delivered to and became accumulated at inflamed joints in 


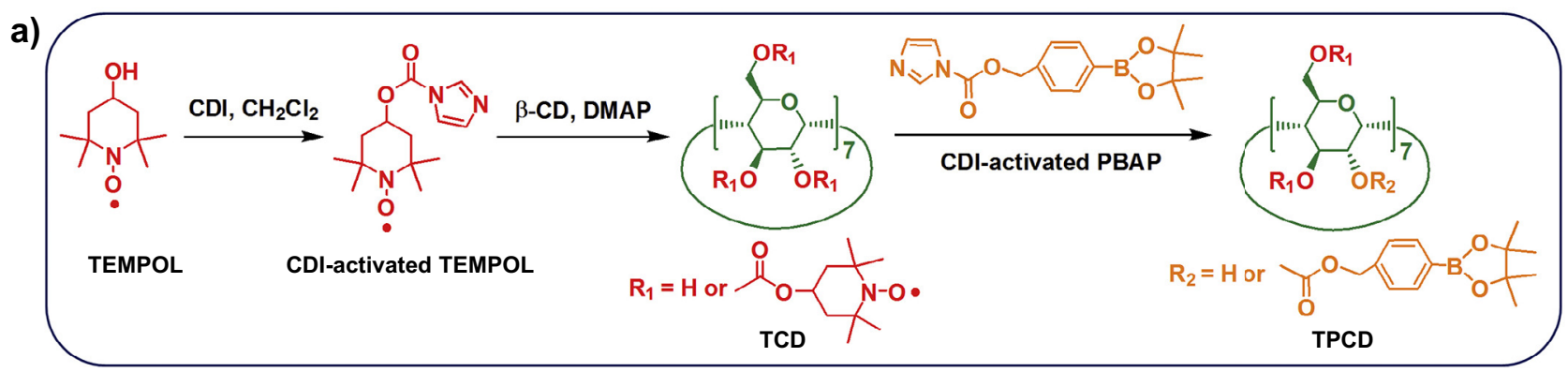

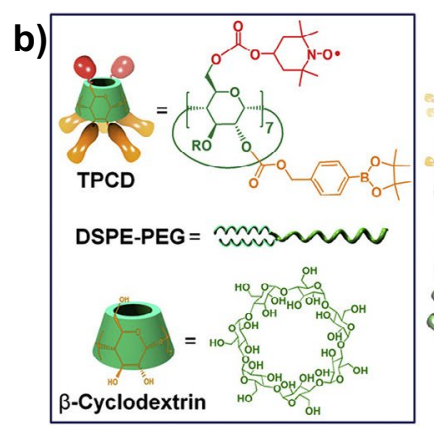

Fig. 1 Construction of broad-spectrum ROS-scavenging nanoparticles. a Synthetic route for $\beta$-CD derivative conjugated with TEMPOL and phenylboronic ester simultaneously. CDI 1,1-carbonyldiimidazole, DMAP 4-dimethylaminopyridine, TCD TEMPOL-conjugated $\beta$-CD, PBAP 4-(hydroxymethyl) phenylboronic acid pinacol ester. b Sche-

collagen-induced arthritis (CIA) mice, enabling the alleviation of joint swelling and cartilage destruction without increased side effects.

Apart from CD derivative-based oxidation-sensitive nanosystems, phenylboronic ester-tethered macromolecules have also been developed for the diagnosis and treatment of chronic inflammatory diseases [42, 43]. For example, Hu and coauthors reported the construction of targeted theranostic nanoplatform encompassing magnetic resonance (MR) imaging-active superparamagnetic iron oxide nanoparticle (SPION) and phenylboronic acid-modified DSPE-PEG [42]. Specifically, Congo red-linked DSPE-PEG (Congo red is capable of binding to amyloid plaques as targeting agent) and phenylboronic acid-modified DSPE-PEG were simultaneously utilized to stabilize oleic acid-coated iron oxide nanoparticles through hydrophobic interactions, followed by drug loading of vicinal diol-containing rutin (a powerful phenolic antioxidant with anti-inflammatory activity) through a dynamic covalent boronate ester linkage [42]. As a result, Congo red/rutin-magnetic nanoparticles coinjected with mannitol could cross the blood-brain barrier (BBB) into the brain of APPswe/PS1dE9 transgenic mice and recognize amyloid plaques, allowing for diagnosis of amyloid plaques by MR imaging and selective delivery of therapeutics. Furthermore, $\beta$-amyloid (A $\beta$ )-induced overproduction of oxidative species could actuate the transformation of phenylboronic ester into phenol and ensuing release of rutin, matic illustration of engineering of a broad-spectrum ROS-scavenging nanoparticle consisting of ROS-reactive $\beta$-CD conjugate, exhibiting oxidation-triggered disintegration. Reproduced with permission from ref. [38]. Copyright 2018, The Authors. Published by Wiley-VCH

resulting in the alleviation of oxidative stress, and reduction of amyloid plaques and neuronal loss.

Alternatively, Jiang et al. presented ROS-responsive polymeric micelles with biomimetic targeting ability to normalize the oxidative and inflammatory microenvironment, and reeducate microglia from an early phase of $\mathrm{AD}$, based on aberrant hyperreactive state of microglia and related microenvironment changes of AD progression [43]. To elaborate, oxidation-triggerable amphiphilic block copolymers (BCPs) were firstly synthesized via post-polymerization modification between hydrophobic phenylboronic ester-containing motifs and amine groups on PEG- $b$-polylysine (PEG- $b$ PLys), followed by conjugation with a small targeting peptide (KLVFFAED; Ab peptide), which is derived from the binding domain of $\mathrm{A} \beta$ protein with receptor for advanced glycation end-products (RAGE) (Fig. 2). Through an A $\beta$ transportation-mimicked pathway, self-assembled, targeted, and curcumin (an immune-modulatory agent with antioxidant and anti-inflammatory capabilities)-loaded micelles could become accumulated into the diseased regions and exert synergistic effects of polymer-based ROS elimination and curcumin-mediated $\mathrm{A} \beta$ inhibition. It should be noted that such multi-target strategy exhibited gradual normalization of brain microenvironment, efficient neuroprotection, and microglia modulation, resulting in the decrease of $\mathrm{A} \beta$ plaque burdens and enhancement of cognitive functions in APPswe/PS1dE9 mice. 


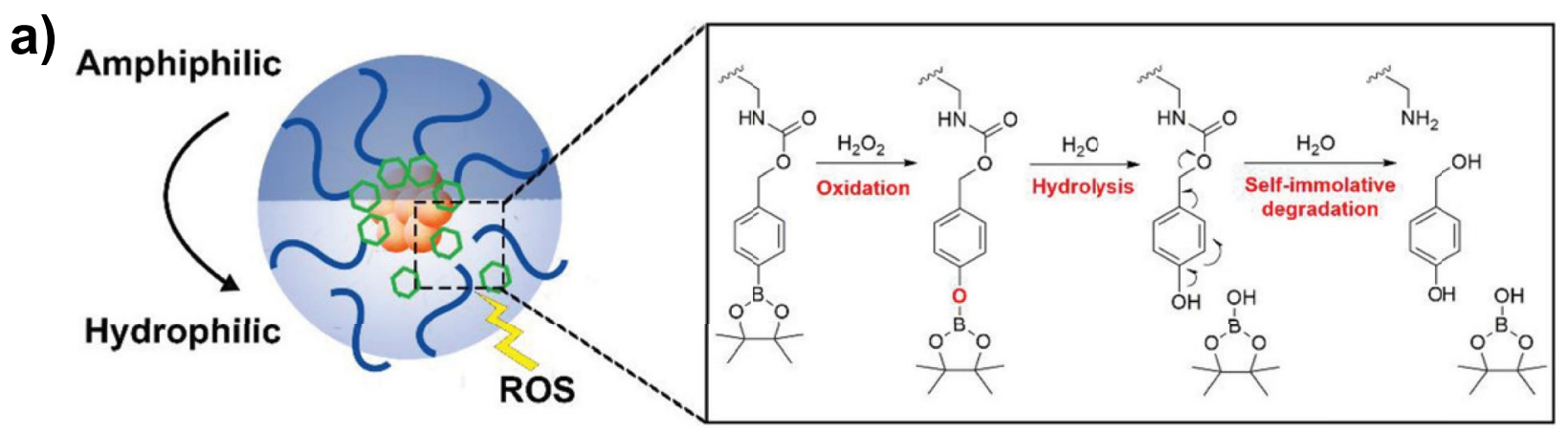

b)

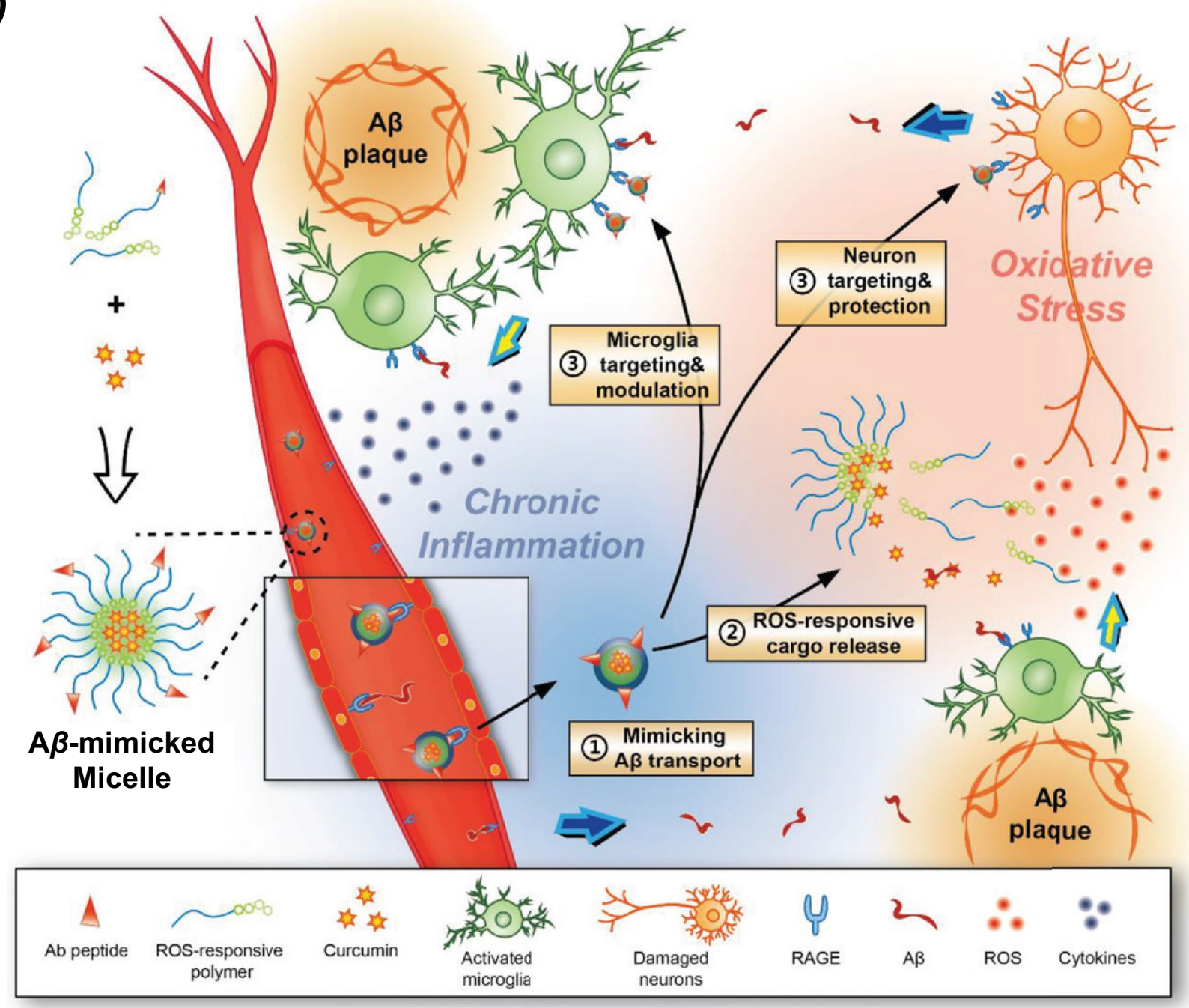

Fig. 2 Oxidation-responsive polymeric micelles with biomimetic targeting capability. a Proposed mechanism of oxidation-actuated selfimmolative side chain degradation of ROS-responsive BCP micelles. b Illustration of microglia induced AD microenvironment and modulation mechanisms of brain-targeting and ROS-responsive micellar delivery system: (i) Ab peptide modified micelles mimic $\mathrm{A} \beta$ transpor- tation from peripheral into brain parenchyma, (ii) ROS-actuated degradation of micelles and triggered release of $\mathrm{A} \beta$ inhibitive curcumin, and (iii) $\mathrm{A} \beta$-mimicked micelle targeting to activated microglia and damaged neurons. Reproduced with permission from ref. [43]. Copyright 2018, The Authors. Published by Wiley-VCH 
On the other hand, recent studies indicate that elevated levels of trace metal ions, especially iron, copper, and zinc ions, are closely interacted with $\mathrm{A} \beta$ aggregate deposition and neurotoxicity in AD [44]. Hence, metal dysregulation has been considered as a promising therapeutic target for AD therapy [45]. However, orthodox metal chelators are not suitable as potential therapeutic agents against $\mathrm{AD}$ due to their inability to efficiently cross BBB and specifically recognize metal ions in various forms [46]. To resolve these problems, Qu et al. developed a mesoporous silica nanoparticle (MSN)-based ROS-responsive system to realize selective delivery of metal chelator $[47,48]$. As a proof of concept, 3-carboxyphenylboronic acid was initially anchored onto MSN surface, followed by conjugation with adjacent diols of saccharides within human IgG through the dynamic boronate ester linkage to trap clioquinol (CQ) inside MSN pores. Such boronate-based and IgG-gated MSN platform was demonstrated as promising DDS for oxidation-triggered delivery of therapeutic metal chelators, showing potential for AD treatment.

Very recently, Lee, Wang, and coauthors reported a biomimetic DDS derived from macrophage membrane-coated chitosan-based nanoparticles with ROS responsiveness for the treatment of atherosclerosis, considering the accumulation of macrophages and increased oxidative stress during the development of atherosclerosis [49]. Notably, surface coating with macrophage membrane not only diminished the clearance of nanoparticles from mononuclear phagocytes system (MPS) but also guided nanoparticles to inflammatory tissues, where the ROS-responsive polymeric scaffold enabled triggered release of encapsulated atorvastatin (AT, a lipid-lowering drug). Moreover, the macrophage membrane was capable of sequestering proinflammatory cytokines and chemokines to achieve local inflammation suppression. The synergetic effects of pharmacotherapy and sequestration of inflammatory cytokines and chemokines from such a biomimetic DDS led to enhanced therapeutic efficiency against atherosclerosis.

\section{Sulfur/selenium-containing systems}

Due to its excellent stability against acid, base, or enzymemediated degradation, thioketal motif that could be efficiently cleaved by diverse oxidants has been increasingly employed for biomedical applications, particularly drug delivery. In 2010, Merlin, Murthy, and coworkers innovatively reported ROS-degradable and thioketal-based polymeric nanoparticles as oral delivery vehicles for small interfering RNA (siRNA), that could direct encapsulated siRNA against TNF- $\alpha$ (TNF- $\alpha$ siRNA) to sites of intestinal inflammation and thus inhibit gene expression in inflamed tissue (Fig. 3) [50]. When delivered orally, such thioketal nanoparticles (TKNs) remained stable in the gastrointestinal tract, protecting TNF- $\alpha$ siRNA against harsh conditions. More importantly, at sites of intestinal inflammation with high levels of ROS, these TKNs selectively underwent ROS-triggered degradation and controlled release of TNF- $\alpha$ siRNA. In a murine model of ulcerative colitis, it was demonstrated that orally administered TNF- $\alpha$ siRNA-loaded nanocomplexes effectively reduced TNF- $\alpha$ messenger RNA (mRNA) levels in the colon and protected mice from ulcerative colitis.

While above-discussed ROS-responsive moieties (i.e., boronate and thioketal) are subjected to cleavage and degradation in response to oxidative milieu, hydrophobic thioether motif undergo oxidation-triggered transformation into hydrophilic sulfoxide or sulfone group [26]. By leveraging this intriguing transformation, Hubbell, Tirelli, and coauthors have elegantly developed a number of oxidationresponsive polymeric nanocarriers (e.g., vesicles and micelles) containing poly(propylene sulfide) (PPS) moieties and these nanocarriers exhibited ROS-actuated solubility switching and destabilization, activating triggered release of a range of encapsulated payloads [26, 51-53]. Likewise, Duvall et al. developed oxidation-sensitive micelles selfassembled from amphiphilic BCPs containing hydrophilic poly ( $N, N$-dimethylacrylamide) (PDMA) and hydrophobic PPS blocks for triggered drug release [54]. It was shown that such destructible fluorophore-loaded micelles efficiently released model drug (e.g., Nile red) upon contact with exogenous $\mathrm{H}_{2} \mathrm{O}_{2}$, $\mathrm{ONOO}^{-}$, or endogenous oxidative species generated inside lipopolysaccharide (LPS)-stimulated RAW264.7 macrophages.

Along the same line, Shuai and colleagues fabricated andrographolide (Andro, a labdane diterpenoid with superior anti-inflammatory activity)-encapsulated oxidationdestructive micellar nanocarriers of PEG- $b$-PPS for the treatment of atherosclerosis via concomitant antioxidative and anti-inflammatory effects [55]. Subsequent detailed evaluations in the mice model of $\mathrm{ApoE}^{-I^{-}}$revealed excellent therapeutic effects and low side effects of Andro-loaded micelles. Very recently, they further explored antirheumatic activity of celastrol (a bioactive component of Tripterygium wilfordii with anti-inflammatory activity)-loaded oxidation-responsive micelles of PEG- $b$-PPS (Fig. 4) [56]. It was demonstrated that such nanomedicine could effectively accumulate in the inflamed joints and subsequently alleviate major RA-related symptoms including synovial inflammation, bone erosion, and cartilage degradation. Analogously, Scott group explored anti-inflammatory potentials of PPSbased nanovehicles with distinct morphologies (i.e., spherical micelles; filamentous worm-like micelles, filomicelles; polymeric vesicles, polymersomes) against atherosclerosis [57-60]. Notably, morphology-dependent organ and cellular level biodistributions of nanocarriers assembled from PEG- $b$-PPS BCPs with identical surface chemistries were disclosed [57]. While spherical micelles and filomicelles 
Fig. 3 Orally delivered TKNs loaded with TNF- $\alpha$ siRNA target inflammation and inhibit gene expression in the intestines. a TNF- $\alpha-$ TKNs were fabricated by first complexing TNF- $\alpha$ siRNA with cationic lipid 1,2-dioleoyl3-trimethylammonium-propane (DOTAP), followed by loading into nanoparticles composed of PPADT. Typical scanning electron microscopy (SEM) image showed TNF- $\alpha$-TKNs (scale bar represents $1.5 \mu \mathrm{m})$. b Orally administrated TNF- $\alpha-$ TKNs remained stable in the harsh conditions of gastrointestinal tract, protecting TNF- $\alpha$ siRNA and preventing its undesirable release to noninflamed mucosal tissues. However, at sites of intestinal inflammation, high levels of ROS triggered degradation of TKNs, thus specifically releasing TNF- $\alpha$ siRNA. Reproduced with permission from ref. [50]. Copyright 2010, Springer Nature

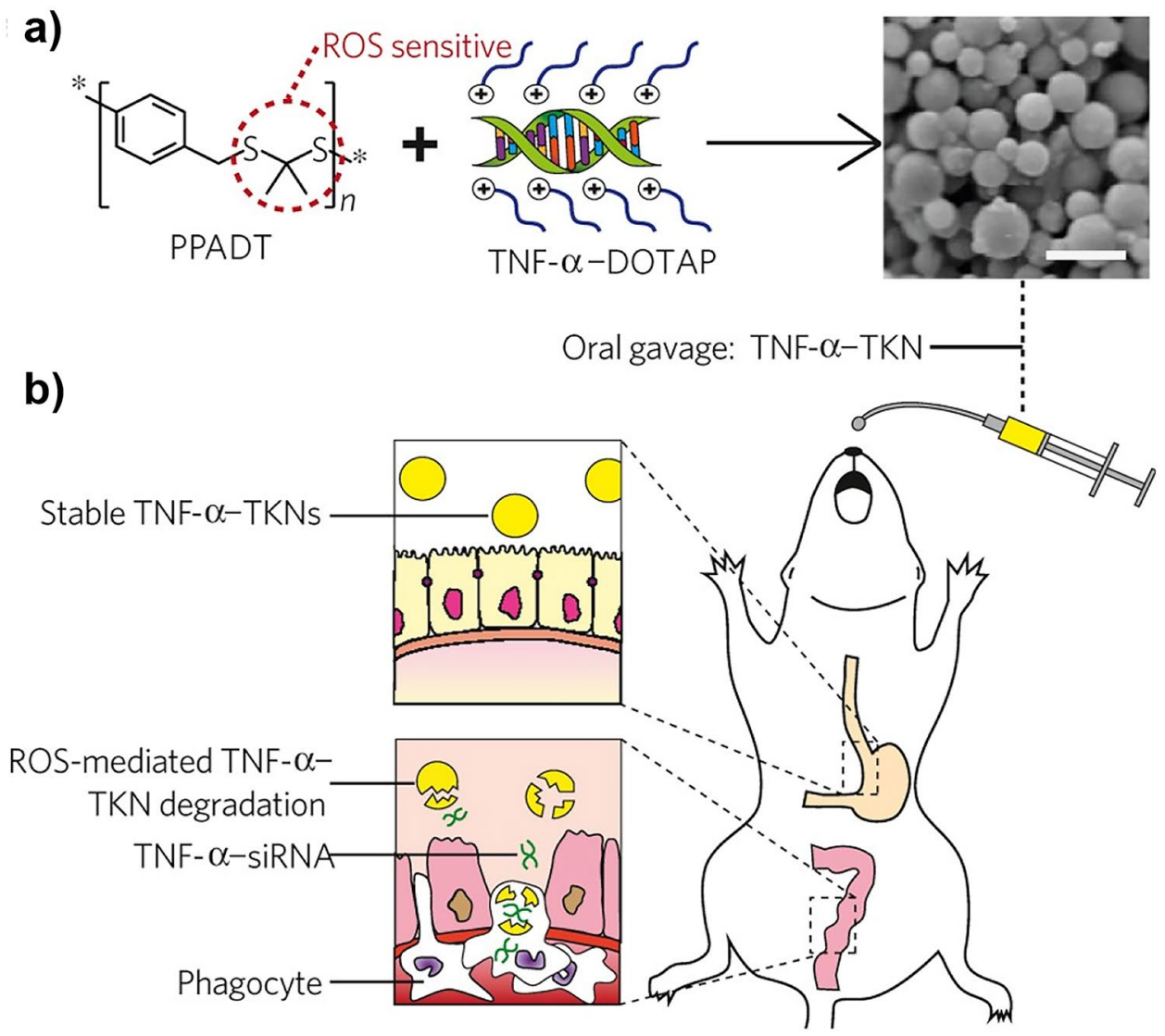

were found to associate with liver macrophages and bloodresident phagocytes, respectively, polymersomes were significantly efficient at targeting splenic dendritic cells (DCs; critical mediators of atherosclerotic inflammation) and exhibited remarkably lower uptake by other cells of the MPS. Furthermore, polymersomes demonstrated superior selectivity for DCs in atherosclerotic lesions in vivo, highlighting the importance of nanostructure morphology in tailoring targeting specificity of nanoscale DDS [57].

Like sulfur-based derivatives, selenium-containing compounds have been widely used in pharmacochemistry as antioxidants, among which diselenide motif constitutes a promising candidate owing to excellent responsiveness towards both oxidative and reductive milieu [61, 62]. In this respect, Liu group reported dually targeting selenium nanoparticles (SeNPs) with antioxidative and neuroprotective activity to inhibit $\mathrm{A} \beta$ fiber formation in $\mathrm{AD}$ [63]. LPFFD and TGN (sequence: TGNYKALHPHNG) peptide cofunctionalized targeted SeNPs efficiently crossed BBB and possessed a strong affinity towards $\mathrm{A} \beta$ species, efficiently suppressing $\mathrm{A} \beta$ fibrillation by disrupting hydrophobic and electrostatic interactions that are involved in the nucleation process of $\mathrm{A} \beta 40$. In addition, it was found that targeted SeNPs could mitigate the $\mathrm{A} \beta 40$ fiber-mediated generation of ROS and neurotoxicity in PC12 cells. In another contribution, Zhang et al. described positively charged polyprodrug amphiphiles to simultaneously encapsulate hydrophobic superparamagnetic iron oxide nanocubes and negatively charged lethal-7b antisense oligonucleotides via hydrophobic and electrostatic interactions, respectively [64]. To elaborate, lipid-lowering drug simvastatin (Sim) was covalently connected to carboxyl-modified zwitterionic poly(carboxybetaine) (PCB) through a diselenide linkage, affording positively charged PCB-Se-Se-Sim with high drug loading content. The formulated MR imaging-traceable nanoparticles could achieve oxidation-actuated corelease of Sim and antisense oligonucleotides to decrease differentiation of exogenous neural stem cells and induce secretion of brain-derived neurotrophic factors concurrently, enabling the proliferation of endogenous neural stem cells for AD treatment.

\section{Oxalate-based systems}

Inspired by the underlying chemiluminescence chemistry of glow sticks, in which oxalate ester selectively decomposes upon contact with $\mathrm{H}_{2} \mathrm{O}_{2}$ to form high-energy and unstable 1,2-dioxetanedione intermediate known to excite nearby fluorophores to release photons, a variety of functional systems containing oxalate moieties and fluorophores have been developed to achieve in vitro and in vivo sensing of ROS 
Fig. 4 Schematic representation of in vivo anti-inflammatory mechanism using ROSresponsive micelles selfassembled from PEG- $b$-PPS for suppressing synovial inflammation and alleviating joint destruction in RA. Reproduced with permission from ref. [56]. Copyright 2020, American Chemical Society
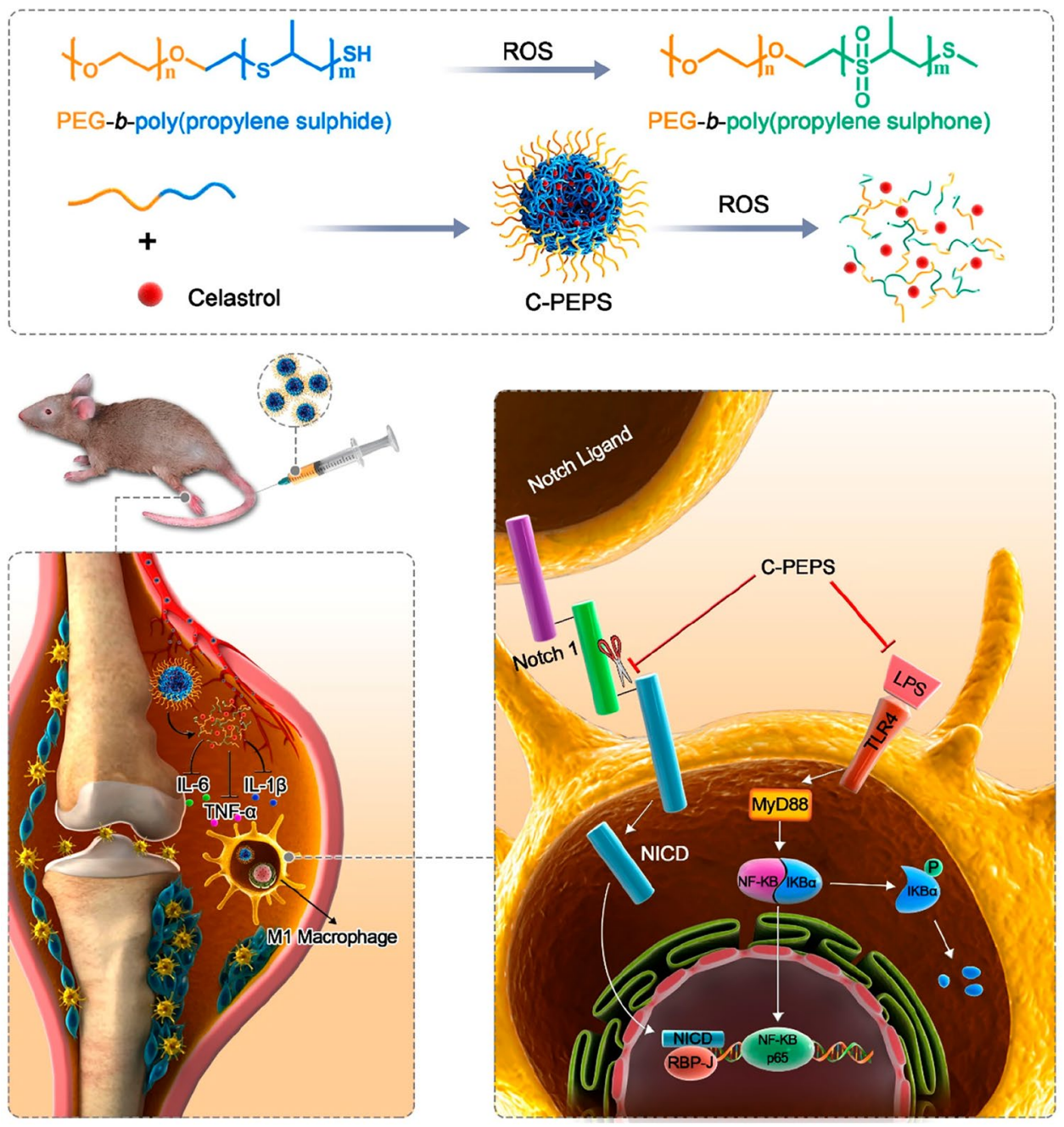

(e.g., $\mathrm{H}_{2} \mathrm{O}_{2}$ ) [65-67]. For instance, Murthy et al. reported the construction of chemiluminescent nanoparticles formulated from oxalate ester-containing polymer and fluorescent dyes and such oxalate-based nanoparticles were capable of imaging $\mathrm{H}_{2} \mathrm{O}_{2}$ with high specificity and sensitivity in the peritoneal cavity of mice during LPS-induced inflammatory response [65]. Subsequently, Lee further explored the antiinflammatory effect of biodegradable polyoxalate nanoparticles featured with ROS-triggered release of $p$-hydroxybenzyl alcohol (HBA) [68, 69]. It was revealed that HBA-backboned copolyoxalate (HPOX) nanoparticles could enable the inhibition of expression of both inducible nitric oxide synthase (iNOS) and production of TNF- $\alpha$ in LPS-activated RAW264.7 macrophage cells. In follow-up works, in vivo anti-inflammatory activities of HPOX nanoparticles with excellent ROS-scavenging capability were investigated as novel therapeutic agents against airway inflammatory diseases $[69,70]$. In a murine model of asthma, intratracheal administration of HPOX nanoparticles substantially reduced the recruitment of inflammatory cells and expression of pro-inflammatory mediators including interleukin-4 (IL-4) and iNOS (Fig. 5) [70].

To further deepen our understanding and improve the clinical translation of nanomedicines, visualization of nanomedicines' fate in vivo, including the circulation, accumulation, and distribution at targeted inflammatory site, is also highly desirable. Towards this end, Ma et al. described a ROS-responsive theranostic nanoplatform for two-photon aggregation-induced emission (AIE) [71] imaging and antiinflammatory therapy of atherosclerosis [72]. Specifically, prednisolone (Pred), a synthetic glucocorticoid agent, was conjugated with a two-photon AIE luminogen (TP) via ROS-cleavable oxalate linkage, forming a theranostic compound TPP (Fig. 6) [72]. Afterwards, TPP was encapsulated into micellar nanoparticles that self-assembled from ROSdisintegrable BCP consisting of hydrophobic thioetherincorporated poly(2-methylthioethanol methacrylate) (PMEMA) and hydrophilic poly(2-methacryloyloxyethyl phosphorylcholine) (PMPC) [72]. Upon oxidative triggering, such theranostic nanomedicine with serial ROS 
Fig. 5 Schematics of HBAbackboned copolyoxalate (HPOX) nanoparticles exhibiting oxidation-actuated degradation and triggered release of antioxidant HBA for the treatment of airway inflammatory diseases. Reproduced with permission from ref. [70]. Copyright 2013, Elsevier

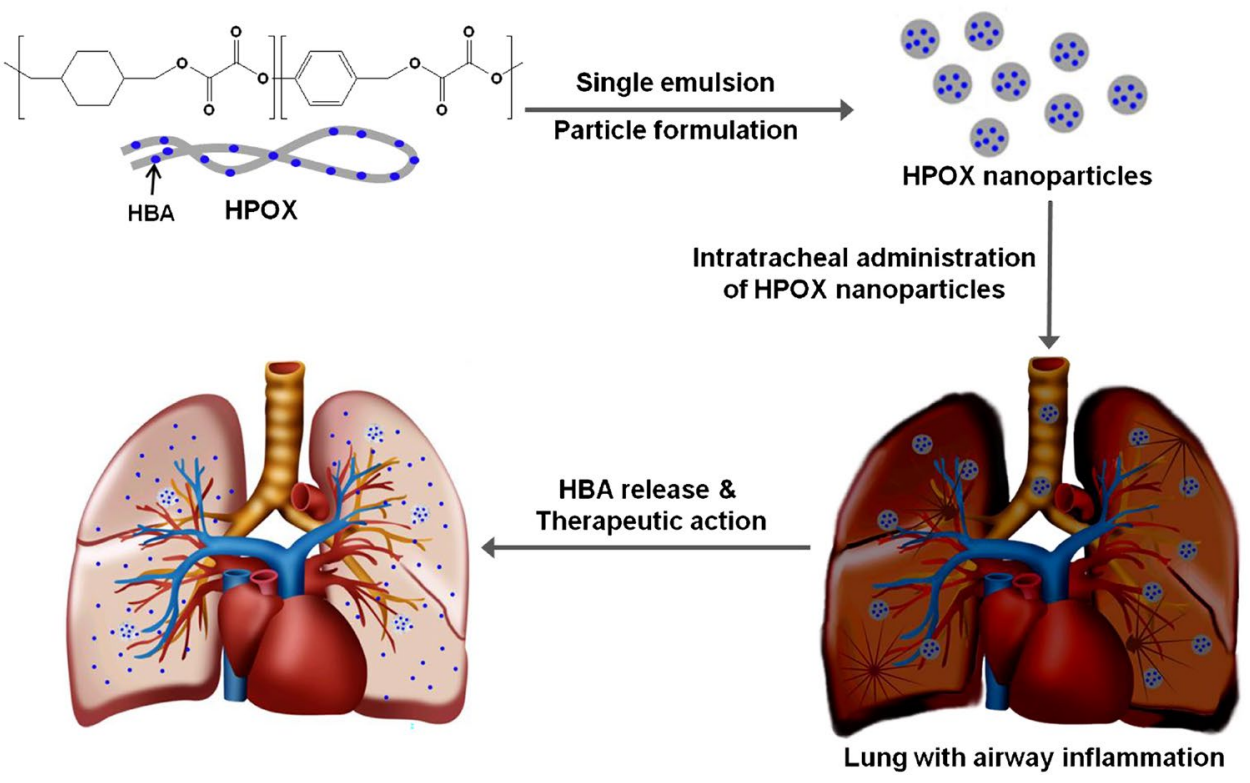

responsiveness firstly underwent ROS-actuated hydrophobic-to-hydrophilic transition and thereby released TPP molecules, followed by ROS-triggered cleavage of oxalate linkage and activation of anti-inflammatory Pred. Moreover, TPP-loaded nanoparticles exhibited strong two-photon fluorescent emission, allowing for a distinct dimensional bioimaging of inflammation site with minimum autofluorescence interference and deep imaging penetration. Importantly, impressive anti-inflammatory activity against RA and atherosclerosis in mice model was discovered through
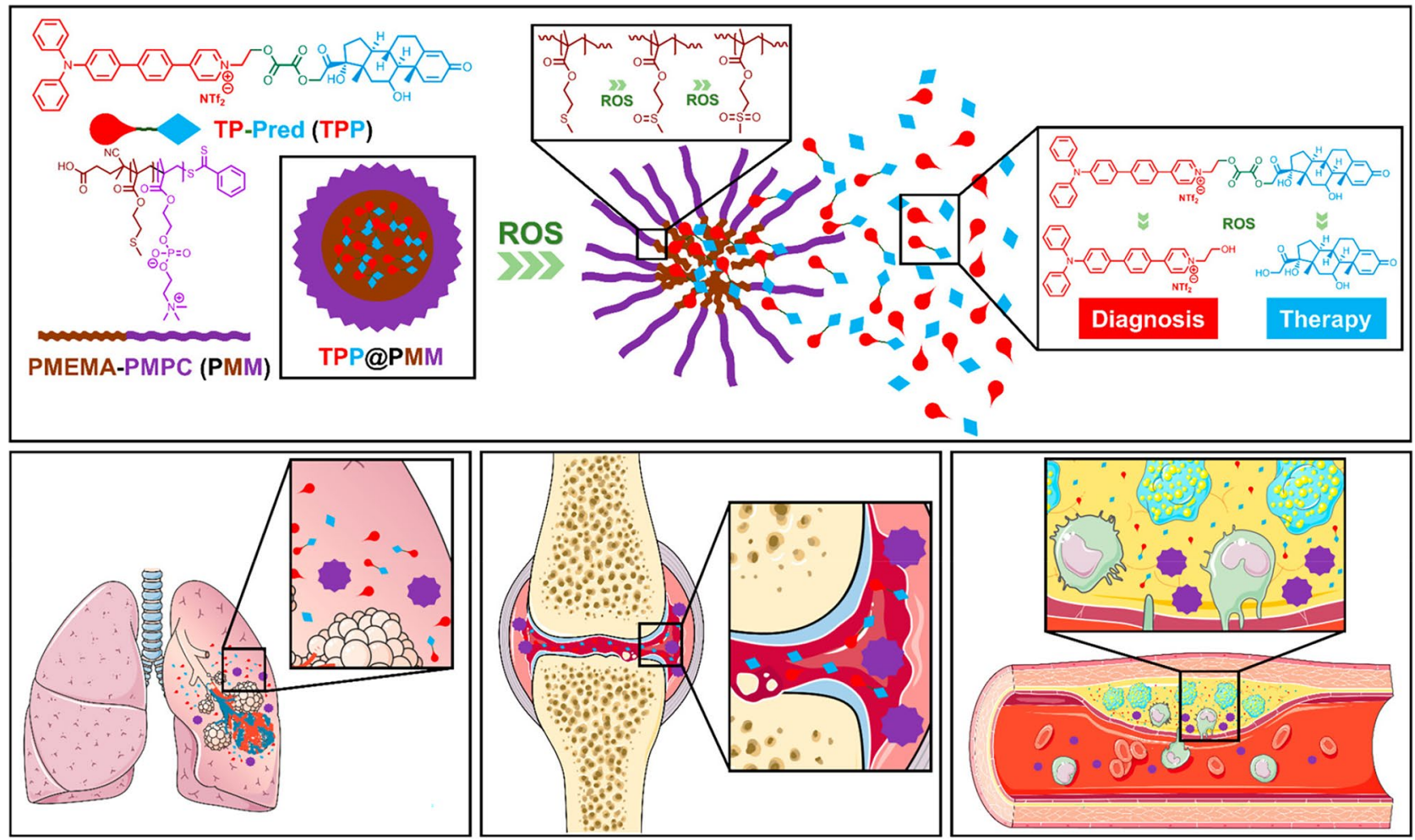

Fig. 6 ROS-responsive theranostic nanoplatform with two-photon imaging capability and anti-inflammatory activity (top). Models of acute lung injury, arthritis, and atherosclerosis were used to evaluate the broad-spectrum inflammation theranostics (bottom). Reproduced with permission from ref. [72]. Copyright 2020, American Chemical Society 
systematic in vivo evaluations, exhibiting reduced inflammatory response and suppressed expression of pro-inflammatory cytokines.

\section{Miscellaneous systems}

Sung and coauthors developed an ultrasensitive ROSresponsive hollow PLGA microcapsule that encompassed an anti-inflammatory dexamethasone sodium phosphate (Dex-P), an acid-generating precursor consisting of ethanol and ferrous chloride $\left(\mathrm{FeCl}_{2}\right)$, and sodium bicarbonate as a gas-generating agent [73]. In cases of inflamed osteoarthritis (OA), low level of $\mathrm{H}_{2} \mathrm{O}_{2}$ could diffuse through the microcapsules to oxidize encapsulated ethanol in the presence of $\mathrm{Fe}^{2+}$ by Fenton reaction, creating an acidic microenvironment. Subsequently, sodium bicarbonate reacted with acid and thus decomposed to form $\mathrm{CO}_{2}$ gas bubbles, disrupting the PLGA shells of microcapsules and releasing high dose Dex-P to the inflamed site, eventually fighting against joint destruction [73].

Bilirubin, as the final product of the metabolism pathway of heme, is a potent endogenous antioxidant capable of scavenging various ROS and thereby protecting cells from oxidative stress-mediated damage. Jon group originally explored anti-inflammatory activity of nanoparticles self-assembled from PEGylated bilirubin for the treatment of colitis, asthma, and psoriasis, by effectively diminishing ROS and modulating the immune system [74-76]. Typically, in a mouse model of ulcerative colitis, intravenous injection of nanoparticles of PEGylated bilirubin exhibited preferred accumulation at the sites of inflammation and remarkably inhibited the progression of inflammation process in the colon [74]. Very recently, $\mathrm{Xie}, \mathrm{Pu}$, and coworkers reported engineered exosomes (Exo) with inflammation-tropism and antiinflammatory effects for concomitant imaging and therapy of atherosclerosis (Fig. 7) [77]. Such Exo were derived from anti-inflammatory M2 phenotype macrophages and further electroporated with FDA-approved hexyl 5-aminolevulinate hydrochloride (HAL), a precursor for biosynthesis of heme. Following systemic administration and localization into inflammatory cells, encapsulated HAL would undergo intrinsic biosynthesis and metabolism of heme to endogenously generate anti-inflammatory carbon monoxide (CO) and bilirubin, which collectively enhanced anti-inflammatory activities and finally alleviated inflammation in atherosclerosis lesions. At the same time, the intermediate product protoporphyrin IX of the heme biosynthesis process allowed for fluorescent imaging and tracking of atherosclerosis.

Concerning the importance of neutrophils and macrophages in inflammatory diseases, regulating inflammatory responses of neutrophils and macrophages with nanomedicine represented a promising approach for disease
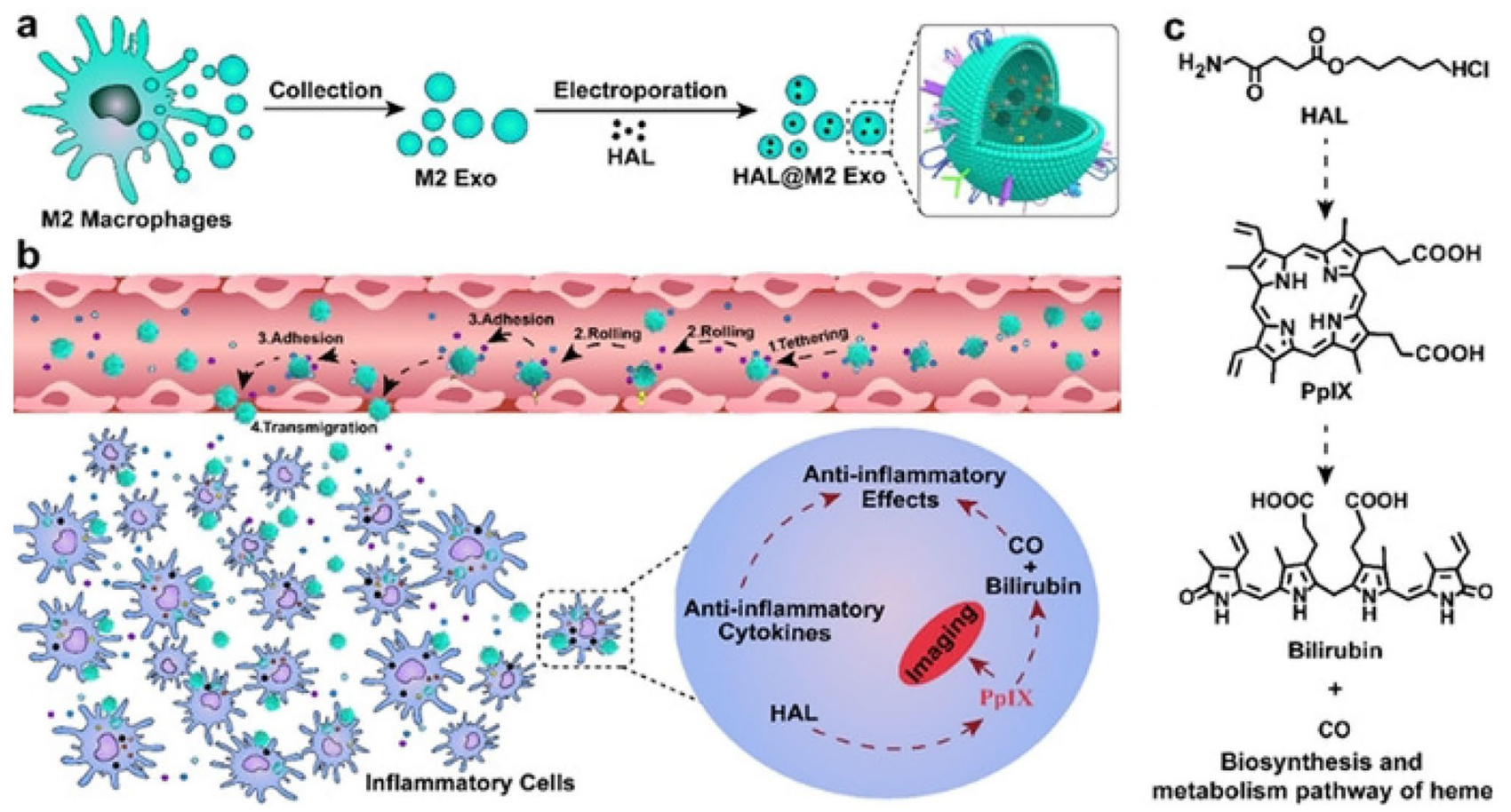

Fig. 7 Engineered macrophage-derived Exo with inflammationtropism and intrinsic heme biosynthesis for atherosclerosis imaging and treatment. a Typical construction route of HAL-encapsulated Exo, HAL@M2 Exo. b The inflammation-tropism and anti- inflammation activity of HAL@M2 Exo. c The intrinsic biosynthesis and metabolism of heme starting from HAL. Reproduced with permission from ref. [77]. Copyright 2019, Wiley-VCH 
management. In this regard, Guo et al. fabricated nanoparticles from luminol-conjugated $\beta$-CD, which efficiently attenuated neutrophil/macrophage-mediated inflammation and demonstrated desirable efficacies and biosafety in treating both acute and chronic inflammatory diseases (e.g., acute lung injury and atherosclerosis) (Fig. 8) [78]. It was found that such bioactive nanoparticles significantly suppressed the production of pro-inflammatory cytokines (e.g., TNF$\alpha$ ) and ROS (e.g., $\mathrm{H}_{2} \mathrm{O}_{2}$ ) by neutrophil/macrophage, and effectively inhibited their migration in vitro. Specifically, in $\mathrm{ApoE}^{-/^{-}}$mice model of atherosclerosis, these nanoparticles efficiently delayed the progression of atherosclerosis as well as notably stabilized atheromatous lesions.

\section{pH-responsive systems}

Local acidification has been recognized at inflammation sites of diverse acute and chronic inflammatory diseases, such as ischemic sites, asthmatic airways, atherosclerotic plaques, and rheumatic joints [7]. Compared to healthy tissues, inflamed tissues generally exhibit lower $\mathrm{pH}$ values [7]. For instance, extracellular $\mathrm{pH}$ in the site of myocardial ischemia was about $6.5-6.0$ and $\mathrm{pH}$ as low as 4.2 was confirmed in the fracture microenvironment $[79,80]$. This mildly acidic characteristic is attributable to enhanced consumption of energy and oxygen by infiltrated inflammationassociated cells, which transform from aerobic metabolism to anaerobic manner inside inflamed tissues, resulting in oxygen-independent glycolysis and increased production of lactic acid $[1,7]$. It should be also mentioned that, in addition to such pathological circumstances, varying degrees of acidic conditions exist in specific cells, tissues, and organs of the human body under normal physiological circumstances. For example, the $\mathrm{pH}$ value along the gastrointestinal tract has a well-tuned gradient, spanning from 1.0 to 3.5 in the stomach to 5.5-7.5 in the intestine [81]. Besides, subcellular compartments display distinct $\mathrm{pH}$ microenvironments, with pH 5.0-6.5 in early and late endosome, $\mathrm{pH} 4.5-5.0$ in lysosome, $\mathrm{pH} 4.0-6.5$ in phagosome, and $\mathrm{pH} \sim 7.8$ within mitochondrial matrix [82]. Collectively, this physiological
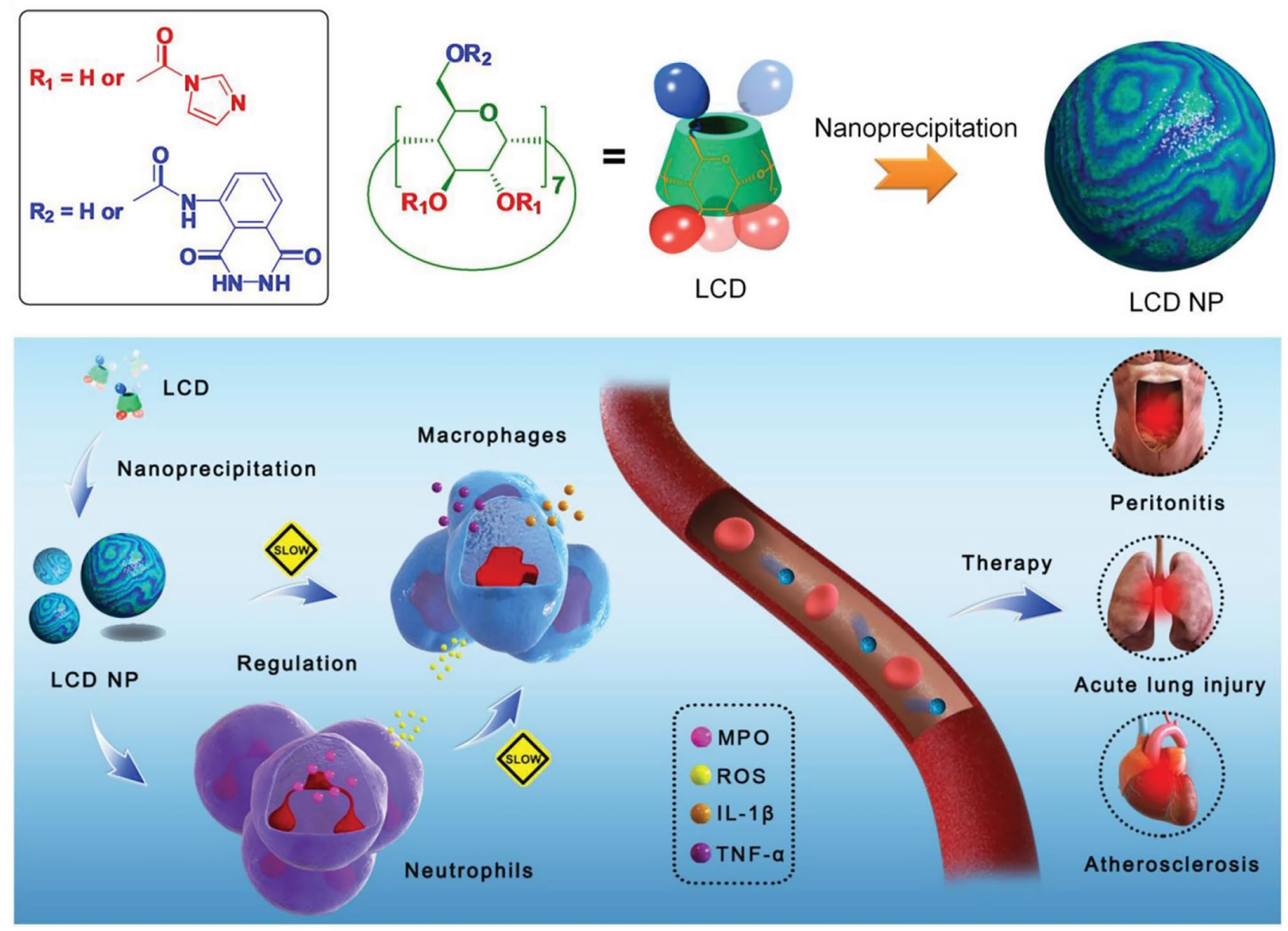

Fig. 8 Development of anti-inflammatory nanoparticles from luminol-modified $\beta$-CD by simultaneously regulating neutrophils and macrophages. Reproduced with permission from ref. [78]. Copyright 2019, Wiley-VCH 
or pathological acidic feature provides a vital biochemical trigger for developing novel $\mathrm{pH}$-responsive DDSs for the management of chronic inflammatory diseases [7, 15].

In 2005, Murthy group reported acid-sensitive polyketal nanoparticles that were designed to transport therapeutic agents (e.g., Dex) to physiologically relevant acidic microenvironment [83]. Such polyketal nanoparticles were fabricated from a hydrophobic polymer, poly (1,4-phenyleneacetone dimethylene ketal) (PPADK). Importantly, these biodegradable polyketal nanoparticles underwent acid-accelerated degradation into low MW and nonacidic products, in contrast to degradation of polyester-based biomaterials. To further address toxicity concern associated with degradation product (i.e., 1,4-benzenedimethanol) of PPADK, they developed novel polyketal, poly(cyclohexane-1,4-diyl acetone dimethylene ketal) (PCADK), with well-documented toxicity profiles [84]. It was found that SOD-encapsulated PCADKbased microparticles enabled efficient scavenging of ROS produced by macrophages in cell culture experiments. Collectively, polyketal-based biomaterials held great potential for treating inflammatory diseases including myocardial infarction, given their neutral, biocompatible degradation products and their capability to deliver a rich assortment of therapeutic agents $[85,86]$. In this aspect, Davis et al. demonstrated that a direct cardiac injection of p38 inhibitor (SB239063)-encapsulated microparticles, fabricated from PCADK, improved cardiac function following myocardial infarction (MI) (Fig. 9) [85]. Significantly, PCADK microparticles induced slight inflammatory responses both in vitro and in vivo and could therefore reside in inflamed site and served as a sustained-release reservoir for SB239063, with a release half-life of 7 days at $\mathrm{pH}$ 7.4. In vivo measurements showed that SB239063-loaded PCADK microparticles exhibited prolonged reduction of inflammatory signaling and eventually increased functional outcome following a single injection, in contrast to nonresponsive PLGA-based system.

Besides ketal-containing systems, acetal-based acidresponsive biomaterials have been extensively explored for numerous biomedical applications [87-90]. For instance, Fréchet et al. elegantly presented an acid-responsive biodegradable nanoparticle from acetal-derivatized dextran with a solubility switching mechanism for controlled delivery of pharmaceutical agents [89]. Using this interesting chemistry, Zhang and coworkers synthesized acetal-modified cyclodextrin and poly(cyclodextrin) with $\mathrm{pH}$-actuated degradability as biocompatible nanomaterials [91]. Water-soluble
Fig. 9 Noninflammatory acidic $\mathrm{pH}$-sensitive microparticlebased delivery system for improved cardiac function following MI. a PCADK microparticles transformed into neutral, nontoxic products through acid-catalyzed hydrolysis of the ketal linkage. b A singleemulsion/solvent-evaporation technique was used to produce large $(\sim 20 \mu \mathrm{m})$ SB239063encapsulated microparticles. Particles were imaged using SEM. c Microparticles were injected intramyocardially, where they released the encapsulated inhibitor within the infarct zone. Reproduced with permission from ref. [85]. Copyright 2008, Springer Nature
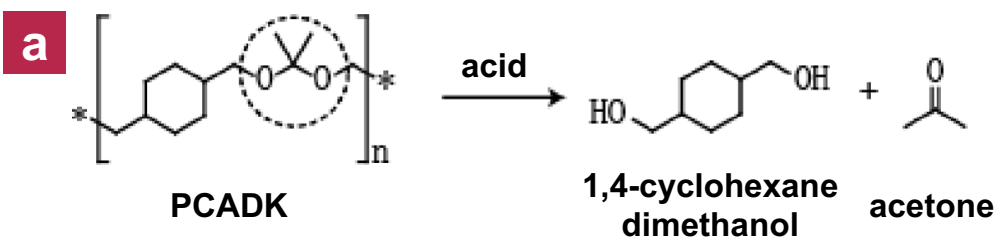

b<smiles>COc1nccc(-c2c(-c3ccc(F)cc3)ncn2C2CCC(O)CC2)n1</smiles>
Single emulsion Solvent evaporation

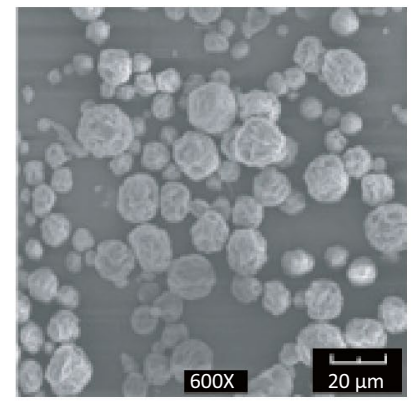

C

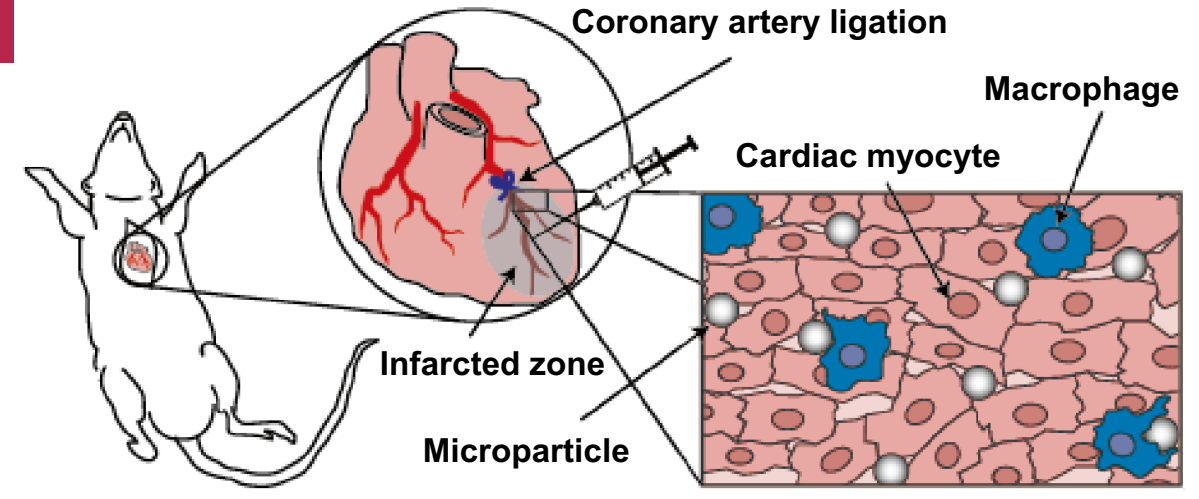


$\alpha$-CD and $\beta$-CD as well as their corresponding condensed polymers were transformed into water-insoluble products through acetalation, respectively. With respect to the formulation process, standard emulsion technique was exploited to prepare nanoparticles with size of about $150 \mathrm{~nm}$. Alternatively, a self-assembling approach was employed to control the sizes and morphologies of assemblies by taking advantage of well-known host-guest interactions (e.g., $\beta$-CD and adamantyl moiety). As expected, nanoparticles from acetalated-CD exhibited acid-triggered hydrolysis, resulting in hydrophobic-to-hydrophilic switching and dissociation of nanoparticles, and thus controlled release of the encapsulated model drug, rapamycin (RAP, an immunosuppressant). More importantly, biocompatibility was further demonstrated for CD derivative-based assemblies via comprehensive measurements both in vitro and in vivo. In their subsequent work, superior targeting ability to atherosclerosis lesions was discovered for acid-responsive nanoparticles of acetalated $\beta$-CD following intraperitoneal injection in $\mathrm{ApoE}^{-/^{-}}$mice [35]. Moreover, in vivo therapeutic outcomes revealed that RAP-loaded nanomedicine from acetalated $\beta$-CD could more efficiently suppress the development of atherosclerosis and effectively improve the stability of atheromatous plaques in comparison with the nonresponsive PLGA-based delivery system.

Wang group developed a series of polymer-drug conjugates (PDCs) with acidic pH-labile acylhydrazone linkage for treatment of diverse chronic inflammatory diseases [92-95]. Typically, $\mathrm{pH}$-sensitive prodrug monomer of Dex was copolymerized with $N$-(2-hydroxypropyl) methacrylamide (HPMA) via reversible additionfragmentation chain transfer (RAFT) polymerization [92]. As expected, the resultant conjugate released Dex upon triggering by acidic milieu ( $\mathrm{pH} 5.0$ ), while remained stable and intact under neutral condition ( $\mathrm{pH}$ 7.4). Notably, in vivo evaluation suggested that such PDCs offered superior, longer-lasting anti-inflammatory activities and joint protection effects when compared to free Dex for the management of RA. Later on, a comprehensive head-to-head comparison study of four established formulations of Dex, including Dex-loaded liposomes (L-Dex), core-crosslinked micelles (M-Dex) with nonselectively cleavable sulfone estercontaining linkage [96], slow releasing PDCs (P-Dex-slow) with acylhydrazone bond, and fast releasing PDCs (P-Dexfast) with acylhydrazone benzyl ester linker, were evaluated in a clinically relevant inflammatory arthritis rat model (Fig. 10) [94]. It was shown that following a bolus intravenous injection, the formulations with slower drug release rates (i.e., M-Dex and P-Dex-slow) provided longer duration of therapeutic activity, i.e., better joint protection, than those with relatively faster drug release kinetics. This finding was instructional and inspiring for the future design, development, and optimization of DDSs for the clinical treatment of RA and other chronic inflammatory diseases. Along this line, Sun et al. reported self-assembling PDCs comprising of PEG-based derivative and hydrophobic anti-inflammatory Pred through cleavable acylhydrazone linker [97]. It was demonstrated that self-assembled polymeric micelles remained intact in circulation for long duration and specifically accumulated in arthritis joints, where the acidic milieu
Fig. 10 Head-to-head comparison study of four established formulations of Dex. a Schematic representation and chemical structures of four dexamethasone-containing nanomedicine formulations. P-Dex-slow and P-Dex-fast encompassed acidic $\mathrm{pH}-$ sensitive linkages for drug conjugation. b Representative microcomputed tomography (micro-CT) images of the ankle joints of Dex-containing nanomedicine-treated adjuvantinduced arthritis rats. P-Dexslow and M-Dex exhibited an impressive reduction of ankle joint bone erosions, where bone was well-preserved. Reproduced with permission from ref. [94]. Copyright 2014, American Chemical Society a)

\begin{tabular}{|c|c|c|c|}
\hline L-Dex & M-Dex & P-Dex-slow & P-Dex-fast \\
\hline & & \\
\hline
\end{tabular}

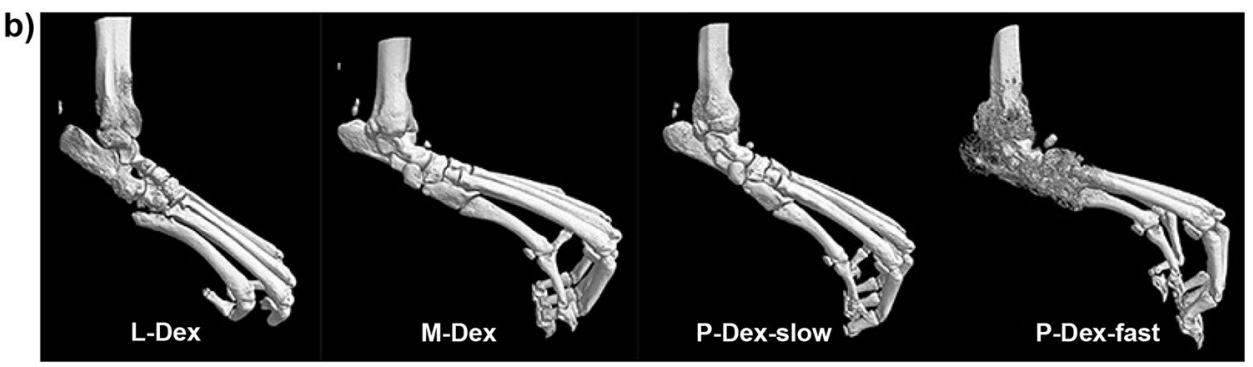


triggered efficient release of Pred, contributing to superior therapeutic efficacy than free Pred against CIA.

As mentioned above, PEGylation has been extensively exploited in DDS to reduce opsonization by proteins and uptake by macrophages of MPS, and prolong plasma residence time following systemic administration [98]. However, after localizing at pathological site, PEGylation might hinder cellular uptake and subsequent endosomal escape, forming an obstacle in the realization of therapeutic activity [99]. To resolve this "PEG dilemma," sheddable PEGylation has been proposed to achieve on-demand dePEGylation at target sites [99]. In this regard, Cui group presented acidic $\mathrm{pH}$-sheddable PEGylated PLGA nanoparticles to improve delivery of therapeutic payloads into chronic inflammation sites [100, 101]. It was demonstrated that hydrazone-based PEGylation of nanoparticles significantly increased the distribution and retention of nanoparticles at pathological lesion when intravenously administered in a mouse model of LPS-induced chronic inflammation [100]. To further augment selective accumulation and/or retention, binding ligand mannose was additionally decorated onto sheddable PLGA nanoparticles to achieve inflammation-associated acidic $\mathrm{pH}-$ triggered conditional targeting [101]. Taken together, acidsensitive sheddable DDSs hold great promise against chronic inflammatory diseases and comprehensive evaluations need to be conducted in vivo in the future.

\section{Enzyme-responsive systems}

\section{Microspheres}

Dated back to 1993, Brown et al. reported an intra-articular polymeric microsphere-based controlled release system consisting of natural polyelectrolytes, gelatin and chondroitin 6-sulfate [102]. The microsphere was fabricated by complex coacervation to encapsulate therapeutic protein, radiolabeled catalase $\left({ }^{14} \mathrm{C}\right.$-catalase $)$. It was shown that kinetics of degradation of microspheres and concomitant release of ${ }^{14} \mathrm{C}$-catalase related to gelatinase activities in the joint fluids. Subsequently, they further optimized the formulation process of microsphere from gelatin and chondroitin 6-sulfate with respect to appropriate loading content and degradation kinetics [103]. In addition, biocompatibility of microsphere-based system was corroborated in human synovial fibroblast culture and by intra-articular administration into the knees of mice. Together, such novel enzyme-degradable, biocompatible, and noninflammatory microsphere delivery system held great potential for the delivery of bioactive proteins to diseased joints (e.g., OA and RA). Annamalai et al. described bioresponsive gelatin microspheres which could be degraded by proteolytic enzymes overexpressed in arthritic flares, leading to spatiotemporally controlled release of anti-inflammatory cytokines for cartilage preservation and repair (Fig. 11) [104]. To elaborate, gelatin microspheres were fabricated by emulsification of solubilized anionic gelatin, followed by covalent crosslinking with amine-reactive genipin. Such negatively charged microspheres were further utilized to sequester cationic cytokines with anti-inflammatory effects (e.g., IL-4 and IL-13) through electrostatic interactions, acting as minimally invasive delivery formulation for OA. Collagenase-triggered degradation of gelatin matrix would result in controlled release of antiinflammatory cytokines, allowing to attenuate the stimulation of chondrocytes and the ensuing secretion of NO.

\section{Hydrogels}

Due to excellent biocompatibility, tunable composition, and tailor-made physiochemical features, hydrogels have found widespread applications in biomedical engineering, such as drug delivery, cell encapsulation, and regenerative medicine [105]. In the context of drug delivery, biodegradable (a)

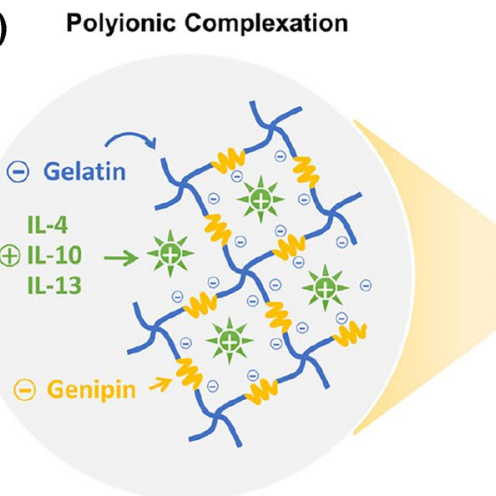

Fig. 11 Enzyme-responsive gelatin microspheres were sensitive to proteolytic enzymes (e.g., collagenase) that typically overexpressed in arthritic flares, resulting in spatiotemporally controlled release of

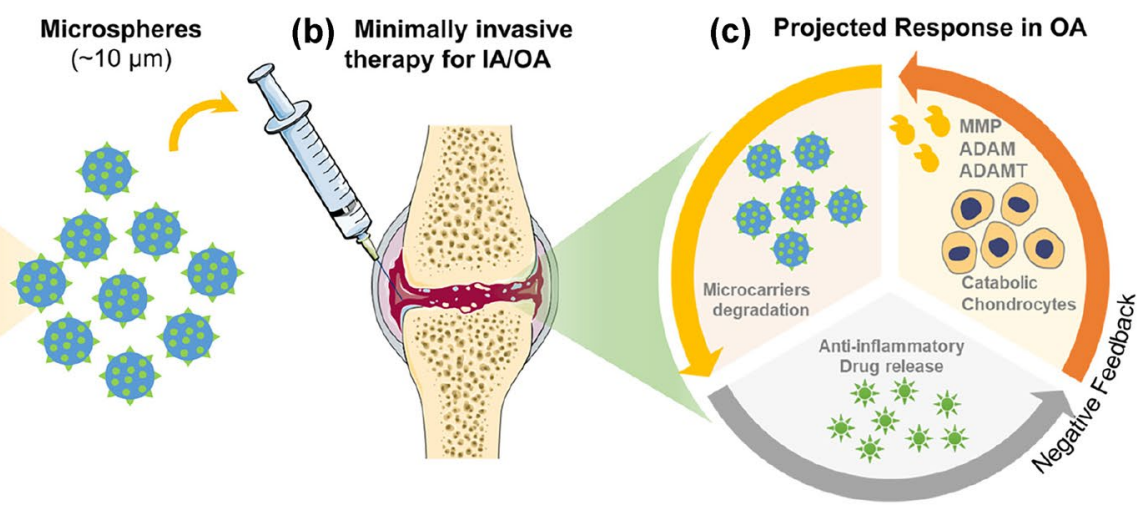

anti-inflammatory cytokines for cartilage preservation and repair in OA. Reproduced with permission from ref. [104]. Copyright 2019, Wiley-VCH 
hydrogels with cleavable backbones, crosslinkers, or sidechains enable release of therapeutic agents in a spatiotemporally controlled manner [105]. In this aspect, Anseth team reported the construction of a proof-of-concept PEG hydrogel with immobilized side-chain, which encompassed human neutrophil elastase (HNE)-cleavable substrate [106]. The kinetic rate of enzyme-mediated cleavage reaction within the hydrogel could be tailored by manipulating the type and sequence of amino acid in substrate, holding promise for cellular responsive drug delivery. Recently, Mejías et al. designed a self-regulated nanoparticle-in-microgel delivery platform, in which microgel decrosslinking was actuated by extracellular HNE, and nanoparticle was loaded with Nexinhib20, a potent inhibitor for neutrophil degranulation [107]. Successful in vivo delivery of Nexinhib20 to the airways and into neutrophils were observed, promoting the resolution of inflammatory response by suppressing recruitment and degranulation of neutrophils, production of pro-inflammatory cytokines in both airway and systemic compartments, as well as neutrophil-derived pathological extracellular vesicles (EVs) in lung fluid.

On the other hand, supramolecular hydrogels formed by low MW gelators that self-assembled in water through multiple noncovalent interactions have garnered considerable attention, representing a promising alternative to polymeric hydrogels [108]. For instance, Xu et al. presented a local delivery system through conjugating tripeptide derivatives with olsalazine, a clinically validated anti-inflammatory prodrug [109]. The resultant amphiphiles self-assembled into nanofibers and then formed supramolecular hydrogels, exhibiting a gel-to-sol transition and controlled generation of bioactive 5-aminosalicylic acid upon azoreductasecatalyzed reduction. In another attempt, Karp and coworkers reported an enzyme-responsive hydrogel platform [110, 111] as inflammation-targeting system for local drug delivery to treat chronic inflammatory diseases $[112,113]$. In an embodiment, hydrogel microfibers capable of encapsulating anti-inflammatory Dex, were generated from amphipathic ascorbyl palmitate containing esterase-cleavable ester linkage [112]. It was demonstrated that these inflammationresponsive hydrogels could exclusively release drug upon enzymatic cleavage and preferentially adhere to inflamed epithelial surfaces both in vitro and in two murine colitis models. In contrast to free Dex enemas, Dex-loaded hydrogel enemas, administered every other day to mice, contributed to a significant attenuation of inflammation and exhibited lower Dex peak serum levels and, thereby, reduced systemic drug exposure. Analogously, the same group

a

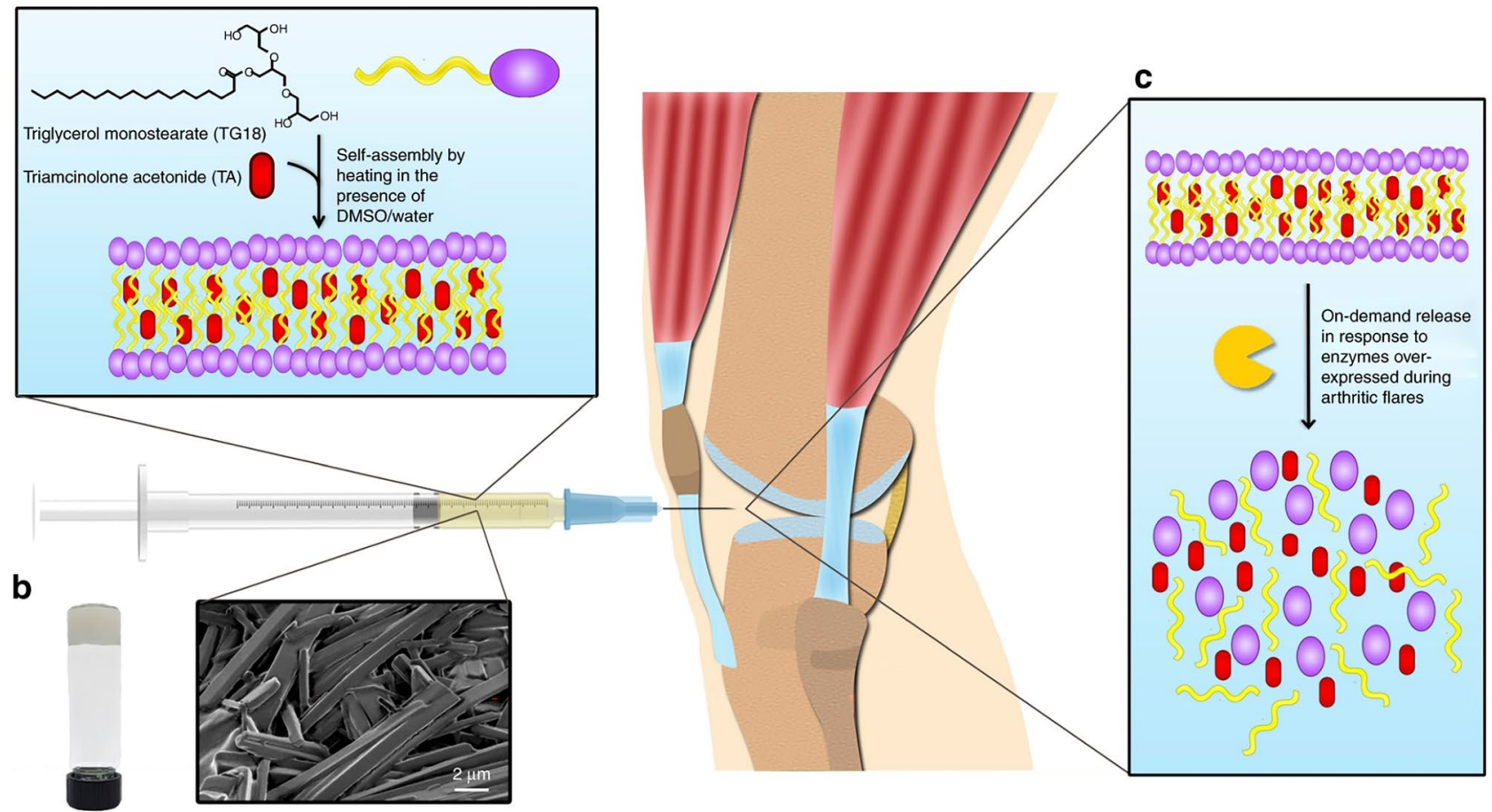

Fig. 12 Arthritis flare-responsive hydrogel platform. a Schematics of self-assembling process of TG-18 to form supramolecular hydrogel and encapsulation of TA. b High-resolution SEM images of TAloaded TG-18 hydrogels (scale bar is $2 \mu \mathrm{m}$ ). TG-18 lamellar structures extended to generate highly ordered fibrous structures. The entangled fibers formed bulk injectable hydrogel that could be administered via intra-articular injection. c Triggered dissociation of TG-18 hydrogel in response to flare-associated overexpressed enzymes (e.g., MMPs) in the inflamed joint. Reproduced with permission from ref. [113]. Copyright 2018, the authors 
developed arthritis flare-responsive hydrogel platform by self-assembling a small-molecule amphiphile, triglycerol monostearate (TG-18), to encapsulate corticosteroid triamcinolone acetonide (TA) (Fig. 12) [113]. It was revealed that TA-loaded hydrogel could achieve on-demand drug release upon contact with enzymes (i.e., esterase or MMPs) or synovial fluid from patients with RA. Notably, a single locally injected dose of TA-encapsulated supramolecular hydrogel suppressed arthritis activity in the injected paw when compared to equivalent dose of free TA.

\section{Polymer-drug conjugates}

By combining the efficacy of two clinically used agents, Homma team reported the construction of hyaluronic acidmethotrexate (MTX) conjugates through varied cathepsinsensitive linkages for the treatment of OA [114, 115]. Through the optimization of linker structure, MW, and stoichiometry of MTX within conjugate, a promising candidate conjugate was identified, capable of inhibiting the proliferation of human fibroblast-like synoviocytes in vitro and knee swelling in rat antigen-induced monoarthritis in vivo [115]. Likewise, several NSAIDs were conjugated onto synthetic dendrimers or linear polymers through enzyme-cleavable linkers in order to alleviate side effects of long-term use of NSAIDs meanwhile retaining their therapeutic activities [116, 117].

\section{Reductive milieu-responsive systems}

In contrast to the mildly oxidizing extracellular environment, the high concentration of glutathione $(1-10 \mathrm{mM})$ and other small redox molecules with free thiol groups (5-10 mM) provide a promising stimulus to devise DDSs for selective intracellular activation [118]. For instance, Xiao et al. presented a strategy that combined the unique property of bioreducible, cationic polymers with superior targeting capability of macrophage-specific mannose residues towards efficient RNA interference (RNAi) for IBD therapy (Fig. 13) [119]. The mannosylated reducible polymer was synthesized via Michael addition reaction between cystamine bisacrylamide (CBA) and branched polyethylenimine (bPEI), and then modified with $D-(+)$-mannose through hydrophilic PEG spacer. TNF- $\alpha$ siRNA-loaded nanosized polyplexes were fabricated through electrostatic interactions, followed by additional stabilization by ionic crosslinker, sodium triphosphate pentabasic (TPP). These polyplexes could efficiently enter macrophages, release encapsulated siRNA upon triggering by reductive milieu, and actuate high-level RNAi, suppressing TNF- $\alpha$ expression and conferring antiinflammatory efficacy in vitro and ex vivo. In another contribution, Zhong, Yin, and coworkers reported a targeted drug/ siRNA codelivery platform based on reversibly disulfidecrosslinked polymersomes for efficient treatment of ulcerative colitis [120].

Apart from reduction-responsive systems for RNAimediated anti-inflammatory therapy, reductive milieusensitive systems have also been explored to transport conventional low MW anti-inflammatory agents and antiinflammatory peptides [121-123]. For instance, Feng and coauthors fabricated reduction-responsive and MTXencapsulated polypeptide-based disulfide-crosslinked nanogels for selective drug delivery in relieving CIA [122]. It was found that such nanogels could selectively accelerate the release of MTX upon responding to GSH to cleave
Fig. 13 Electrostatic interactiondriven self-assembling process into mannose-functionalized, siRNA-encapsulated nanoparticles for macrophage-targeting delivery and cytosolic reductive milieu-triggered release of siRNA. Reproduced with permission from ref. [119]. Copyright 2013, Elsevier Ltd

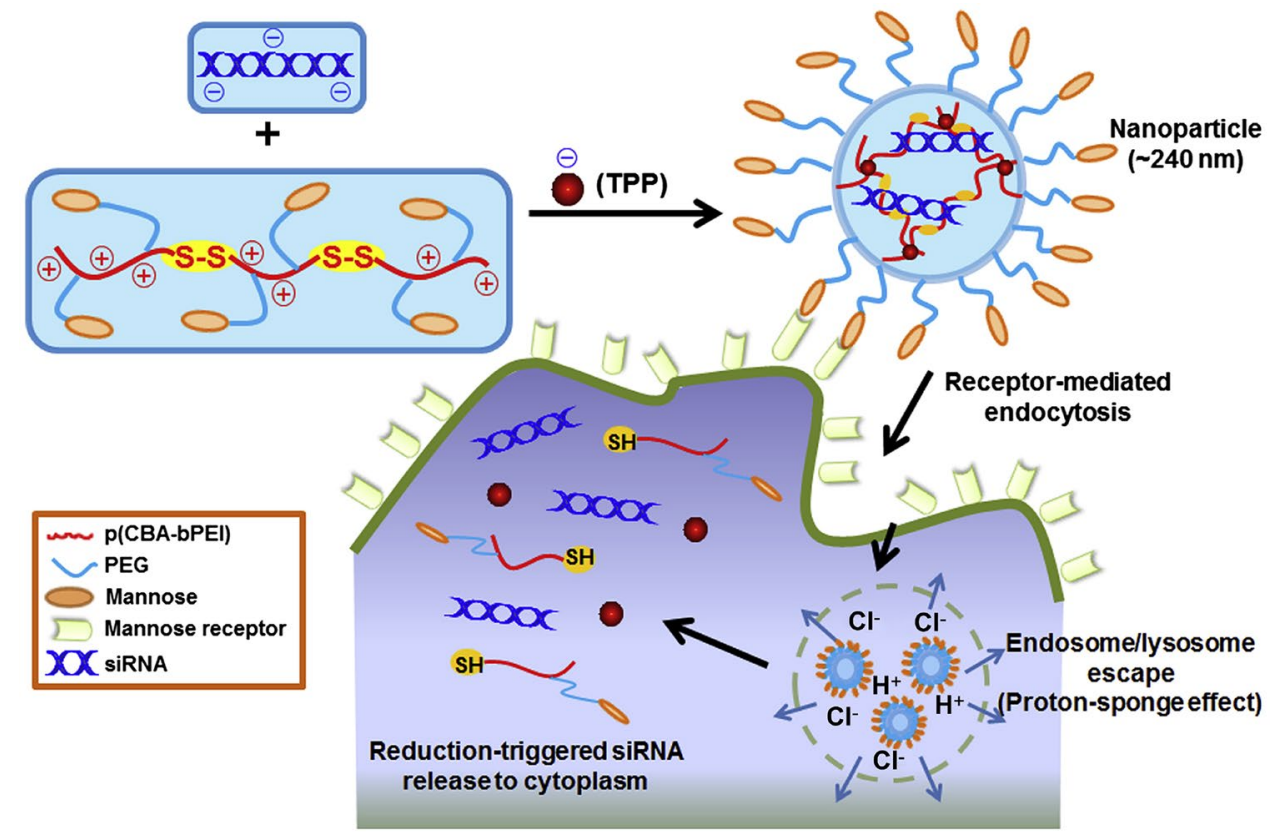


disulfide-based crosslinkers. Moreover, drug-loaded nanogels exhibited selective accumulation in the inflammatory joints of CIA mouse model, effectively inhibiting secretion of proinflammatory cytokines and relieving the progress of CIA.

\section{Multi-stimuli-responsive systems}

Because of the large variations in physiological conditions between normal tissues and diseased sites, smart materials with single responsiveness may not achieve the desired goals in a complex pathophysiological microenvironment [124-126]. Instead, materials responsive to multiple physical, chemical, and biological stimuli are highly promising for advanced biomedical applications, including precision drug delivery [124-126]. As discussed above, oxidative stress, acidic $\mathrm{pH}$, and overexpressed enzymes are important pathological stimuli for drug delivery to inflamed sites. Towards developing multi-stimuli-responsive systems, Almutairi group presented an innovative approach to devise inflammation-responsive logic gate nanoparticles for triggered delivery of proteins [127]. Specifically, they constructed an oxidation and acidic $\mathrm{pH}$ dually responsive polythioether ketal by integrating a thioether moiety in $\mathrm{pH}$-responsive polyketal backbone; oxidation-reactive thioether acted as a solubility switch that turned the polymer more hydrophilic when it was subjected to oxidation [51,52]. Higher release kinetics of encapsulated ovalbumin was observed in the presence of both $\mathrm{H}_{2} \mathrm{O}_{2}$ and acidic $\mathrm{pH}$. In another report, $\mathrm{Zhu}$ and coworkers constructed polydatin (PD; a bioactive agent with hepatoprotective and antifibrotic activity)-loaded micelles assembled from ROS and acidic pH dually sensitive BCPs to ameliorate liver fibrosis [128]. The PD-encapsulated micelle exerted great promise in improving the biocompatibility of PD and exhibited highly effective liver-targeted drug delivery in response to the fibrotic microenvironment. Both in vitro and in vivo evaluations demonstrated that PD-loaded micelles could significantly suppress inflammatory response and oxidative stress, reduce hepatocyte apoptosis, and avert activation of macrophages and hepatic stellate cells, endowing them with remarkable efficacy and minimal side effects in the management of liver fibrosis. In an alternative manner, Lee et al. developed an oxidation and acid dually responsive antioxidant polymeric prodrug of vanillin, poly(vanillin oxalate) (PVO), in which anti-inflammatory activity of vanillin was blocked via $\mathrm{H}_{2} \mathrm{O}_{2}$-reactive peroxalate ester and acidresponsive acetal linkages [129]. It was revealed that PVO nanoparticles exhibited highly potent antioxidant effects by scavenging ROS and inhibiting the production of ROS, as well as suppressed the expression of pro-inflammatory chemokines and cytokines in activated macrophages in vitro and in vivo. Analogously, Daniel and coauthors reported a proof-of-concept example of dual-responsive nanoparticles capable of activating bioactive MMP inhibitor (designated as PY-2) upon contact with MMP and ROS [130]. Exposure of such dually responsive micellar nanoparticles to MMP-12 induced a morphological transformation into micron-scale aggregates, which was beneficial for enhanced retention of nanomedicine in targeted lesions [131, 132]. This change together with the presence of $\mathrm{H}_{2} \mathrm{O}_{2}$, resulted in controlled release of PY-2, potentially relieving inflammation by inhibiting enzymatic activities of MMPs.

Sung et al. described a blended nanoparticle system with dual responses to oxidative stress and reduced $\mathrm{pH}$ through coassembling pH-responsive Cy3-conjugated $\mathrm{N}$-palmitoyl chitosan and ROS-degradable poly-(1,4-phenyleneactone dimethylene thioketal) (PPADT) for delivery of curcumin (Fig. 14) [133]. Notably, owing to the presence of Förster resonance energy transfer (FRET) between curcumin and conjugated fluorophore $\mathrm{Cy} 3$, such system enabled in situ monitoring of intracellular release behavior. Furthermore, in vivo anti-inflammatory effects of curcumin-loaded nanoparticles were validated in a mouse model with ankle inflammation induced by LPS, revealing significant mitigation of oxidative stress. Along this line, reactive oxygen species (ROS)-sensitive dextran-naproxen conjugate was blended with acid-responsive acetalated dextran polymer, forming a dually responsive nanoparticle with high specificity towards inflammatory microenvironment [134].

In another comprehensive work, a facile and effective approach was proposed to tailor $\mathrm{pH} /$ oxidation dual-responsive nanocarriers by combination of $\mathrm{pH}$-sensitive acetalated $\beta$ $\mathrm{CD}$ and oxidation-responsive phenylboronate conjugated $\beta$ $\mathrm{CD}$ [135]. By tuning the weight ratio of acidic $\mathrm{pH}$-sensitive material and ROS-responsive material, coassembled nanoparticles with desirable $\mathrm{pH} /$ oxidation-responsive capability could be easily produced. In response to reduced $\mathrm{pH}$ or oxidative stress, encapsulated RAP molecules could be released from drug-loaded dually responsive nanoparticles. Furthermore, targeting capacity of such dual-responsive nanoparticles was enhanced by surface decoration with a type IV collagen targeting peptide (sequence: KLWVLPKGGGC), resulting in an actively targeted nanoplatform. Accordingly, RAP-loaded targeted nanoparticles displayed more effective in vivo efficiency than passively targeted nanoparticles in the inhibition of neointimal formation in rats. Taken together, these developed $\mathrm{pH} /$ oxidation dualresponsive nanocarriers held great promise for the management of restenosis and other cardiovascular diseases related to vascular inflammation.

On the other hand, Santos group presented $\mathrm{pH}$ and oxidation sequentially responsive nano-in-micro composites for targeted therapy of IBD [136]. Specifically, oxidation-sensitive nanoparticles formulated from a phenylboronic esters-modified dextran were coated with mucoadhesive chitosan, followed by encapsulation in $\mathrm{pH}$-responsive hydroxypropyl 


\section{Composition of Nanoparticles}

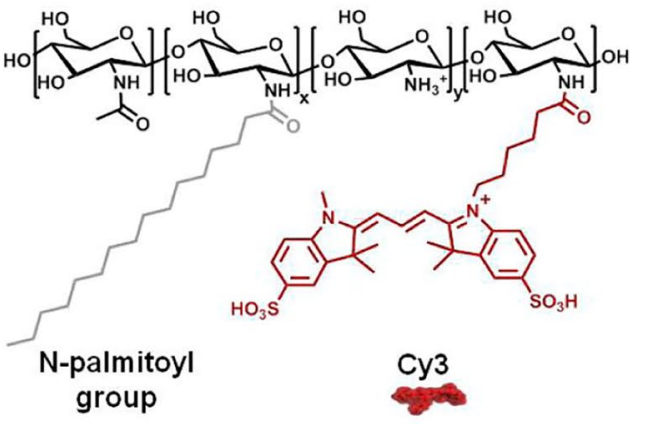

\section{Cy3-conjugated $\mathrm{N}$-palmitoyl chitosan} (Cy3-NPCS)

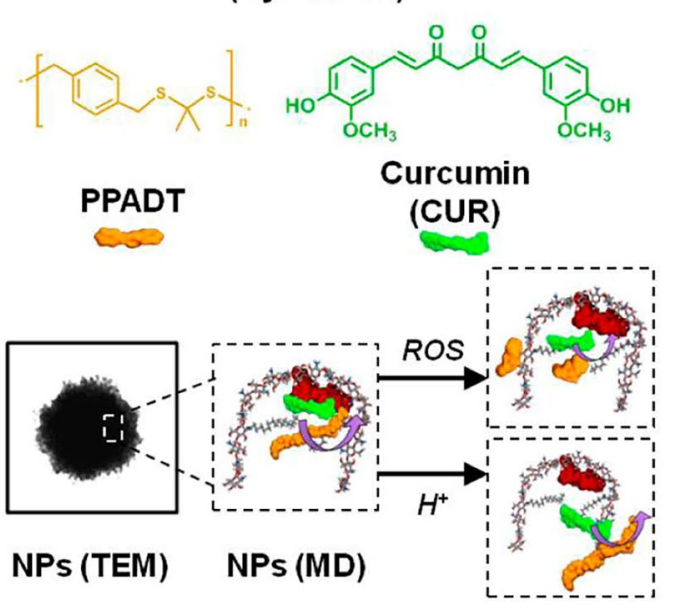

\section{Anti-inflammatory Mechanisms}
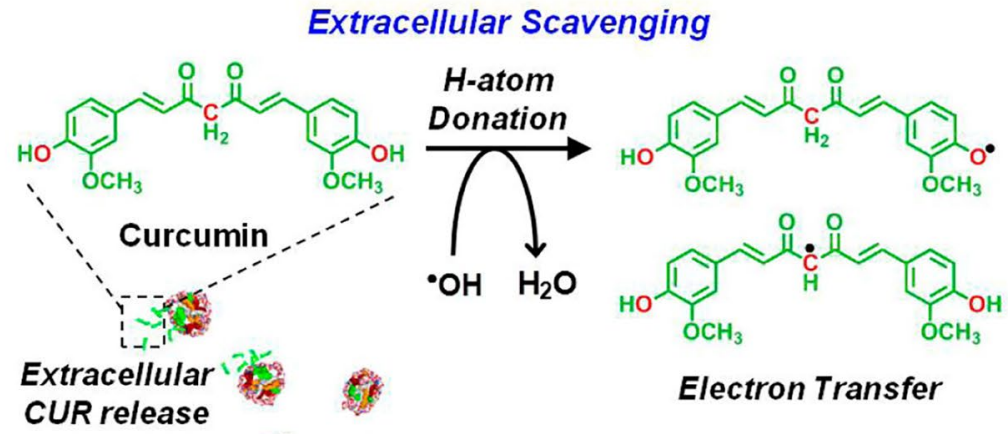

Electron Transfer

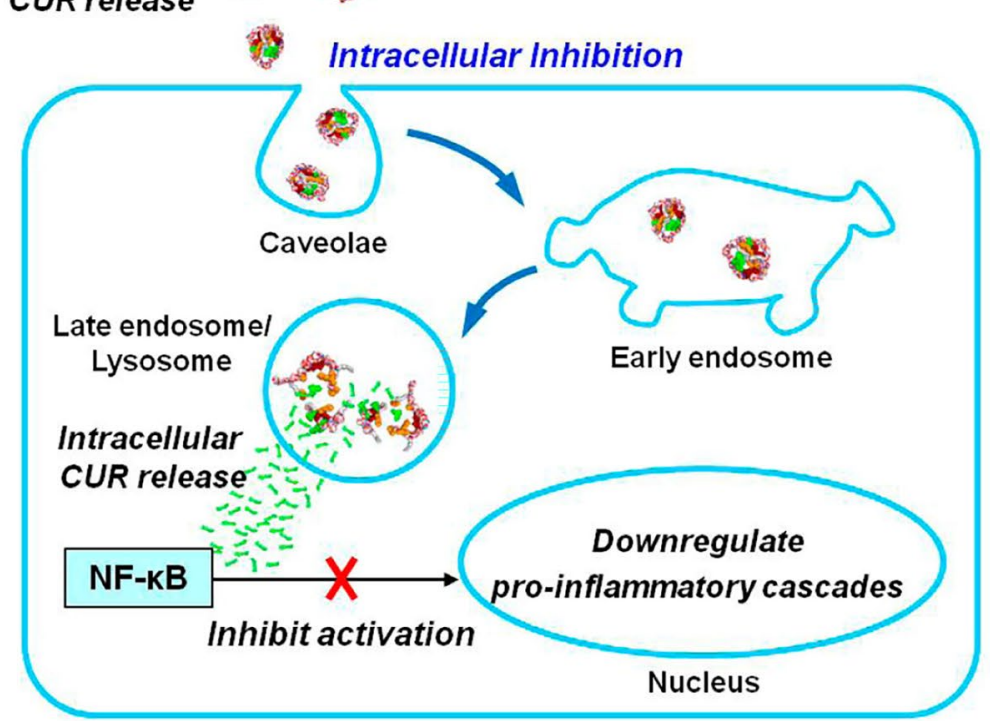

Fig. 14 Schematic illustrations showing the composition and structure of oxidation and acidic $\mathrm{pH}$ dually responsive nanoparticles and their extracellular/intracellular anti-inflammatory mechanisms.
Reproduced with permission from ref. [133]. Copyright 2014, American Chemical Society

\section{Conclusions} fluidics technique, affording nano-in-micro composite particles. The final composites were devised to protect the rifaximin (RIF; an intestine-specific antibiotic employed in inducing remission of IBD)-loaded oxidation-responsive nanoparticles from the harsh conditions in the upper gastrointestinal tract (e.g., acidic $\mathrm{pH}$ of the stomach) and to unmask them in the intestine, closer to the targeted lesions. Upon activating by elevated ROS levels, oxidation-responsive nanoparticles would selectively release RIF into the inflamed sites. Unfortunately, comprehensive in vivo anti-inflammatory evaluations of such promising nano-in-micro composites have not been systematically investigated.
In this review, recent progress on the development of inflammation-responsive delivery systems as promising anti-inflammatory therapy in the treatment of chronic diseases is summarized. The rational design of intelligent DDSs enables selective, on-demand release and activation of bioactive agents at target disease sites by leveraging inflammation-associated biochemical signals, including oxidative stress, reduced $\mathrm{pH}$, overexpressed enzymes, and reductive microenvironment. In vivo antiinflammatory potentials of inflammation-sensitive DDSs have been preliminarily demonstrated via local, oral, or 
systemic administration in diverse animal models, exhibiting enhanced efficiency as compared to nonresponsive systems. Notwithstanding these advances, only a very few inflammation-responsive DDSs have been comprehensively explored in various animal models of chronic inflammatory diseases. Even worse, excellent anti-inflammatory effects of inflammation-sensitive delivery systems in animal models of chronic inflammatory diseases have not progressed in a clinical trial phase. To our knowledge, no translational studies have been carried out in inflammationresponsive therapies towards the treatment and management of chronic diseases [8, 15]. In contrast, several traditional and nonresponsive anti-inflammatory DDSs have succeeded to reach clinical trials [8]. This status is reminiscent of the development process of smart DDSs in cancer treatment [137], challenging the therapeutic benefits of bioresponsive delivery systems over traditional systems in clinical applications.

Numerous challenges in this burgeoning field remain to be addressed in the future before clinical translation studies. First, for anti-inflammatory therapy, diverse biological barriers impede the selective accumulation of DDSs and therapeutic agents at the site of inflammation, compromising in vivo therapeutic efficiency and benefit-to-risk window [1]. Second, new and standard preclinical animal models that could thoroughly mimic the pathophysiological characteristics of human chronic inflammation need to be established. Thus far, most in vivo therapeutic studies of inflammation-responsive DDSs were conducted in murine models of chronic inflammatory diseases, and the collected results could not be directly correlated to humans. Third, for rational design and development of inflammation-responsive DDSs, the optimal biochemical stimuli should be determined according to disease types and diseased sites. For instance, the extent of oxidative stress and the major type of oxidative species might be dynamically changed in distinct chronic diseases, even at different stages of one disease. While multi-stimuliresponsive systems theoretically could provide better sensitivity and selectivity, their formulations generally involve complicated synthesis and/or fabrication procedures. Last but not least, further work is needed to surmount pharmaceutical barriers ranging from biocompatibility and safety of various DDSs, cost of goods and manufacturing, to batch-to-batch reproducibility, facilitating potential clinical translations of inflammation-sensitive delivery systems.

Acknowledgements The authors would like to acknowledge the support from University of Science and Technology of China, Hefei, Anhui Province, China.

Author contribution Writing-original draft preparation: Zhengyu Deng. Writing-review and editing: Shiyong Liu.
Funding This work was supported by National Key Research and Development Program of China (2020YFA0710700) and National Natural Science Foundation of China (NNSFC) Project (51690150, 51690154, 52021002, 21925107, and U19A2094).

Data availability Data sharing is not applicable to this article as no datasets were generated or analyzed during the current study.

\section{Declarations}

Research involving human and animals This article does not contain any studies with human participants or animal models performed by any of the authors.

Consent for publication The authors declare that we participated in the development of this manuscript and have approved the final version, and give our consent for this submitted manuscript to be considered in Drug Delivery and Translational Research.

Competing interests The authors declare that they have no conflict of interest.

\section{References}

1. Tabas I, Glass CK. Anti-inflammatory therapy in chronic disease: challenges and opportunities. Science. 2013;339(6116):166-72.

2. Netea MG, et al. A guiding map for inflammation. Nat Immunol. 2017;18(8):826-31.

3. Bennett JM, Reeves G, Billman GE, Sturmberg JP. Inflammationnature's way to efficiently respond to all types of challenges: implications for understanding and managing "the epidemic" of chronic diseases. Front Med. 2018;5:316.

4. Schett G, Neurath MF. Resolution of chronic inflammatory disease: universal and tissue-specific concepts. Nat Commun. 2018;9(1):3261.

5. Dinarello CA. Anti-inflammatory agents: present and future. Cell. 2010;140(6):935-50.

6. Crielaard BJ, Lammers T, Schiffelers RM, Storm G. Drug targeting systems for inflammatory disease: one for all, all for one. $\mathrm{J}$ Controlled Release. 2012;161(2):225-34.

7. d'Arcy R, Tirelli N. Fishing for fire: strategies for biological targeting and criteria for material design in anti-inflammatory therapies. Polym Adv Technol. 2014;25(5):478-98.

8. Brusini R, Varna M, Couvreur P. Advanced nanomedicines for the treatment of inflammatory diseases. Adv Drug Del Rev. 2020;157:161-78.

9. Matsumura Y, Maeda H. A new concept for macromolecular therapeutics in cancer chemotherapy: mechanism of tumoritropic accumulation of proteins and the antitumor agent Smancs. Cancer Res. 1986;46:6387-92.

10. Maeda H. Macromolecular therapeutics in cancer treatment: the EPR effect and beyond. J Controlled Release. 2012;164(2):138-44.

11. Wang D, et al. The arthrotropism of macromolecules in adjuvant-induced arthritis rat model: a preliminary study. Pharm Res. 2004;21(10):1741-9.

12. Wang D, Miller SC, Liu XM, Anderson B, Wang XS, Goldring SR. Novel dexamethasone-HPMA copolymer conjugate and its potential application in treatment of rheumatoid arthritis. Arthrit Res Ther. 2007;9(1):R2. 
13. Kushner I, Somerville JA. Permeability of human synovial membrane to plasma proteins. Relationship to molecular size and inflammation. Arthritis Rheum. 1971;14(5):560-70.

14. Espinosa-Cano E, Aguilar MR, Vázquez B, San Román J. Inflammation-responsive polymers. In: Aguilar MR, San Román J, editors. Smart Polymers and their Applications (Second Edition). Woodhead Publishing; 2019. p. 219-54.

15. Dou Y, Li C, Li L, Guo J, Zhang J. Bioresponsive drug delivery systems for the treatment of inflammatory diseases. J Controlled Release. 2020;327:641-66.

16. Khutoryanskiy VV, Tirelli N. Oxidation-responsiveness of nanomaterials for targeting inflammatory reactions. Pure Appl Chem. 2008;80(8):1703-18.

17. Lobatto ME, Fuster V, Fayad ZA, Mulder WJ. Perspectives and opportunities for nanomedicine in the management of atherosclerosis. Nat Rev Drug Discov. 2011;10(11):835-52.

18. Maruf A, et al. Atherosclerosis treatment with stimuli-responsive nanoagents: recent advances and future perspectives. Adv Healthcare Mater. 2019;8(11):e1900036.

19. Zhang S, Langer R, Traverso G. Nanoparticulate drug delivery systems targeting inflammation for treatment of inflammatory bowel disease. Nano Today. 2017;16:82-96.

20. Park JH, Dehaini D, Zhou J, Holay M, Fang RH, Zhang L. Biomimetic nanoparticle technology for cardiovascular disease detection and treatment. Nanoscale Horizons. 2020;5(1):25-42.

21. Deng Z, Hu J, Liu S. Reactive oxygen, nitrogen, and sulfur species (RONSS)-responsive polymersomes for triggered drug release. Macromol Rapid Commun. 2017;38(11):1600685.

22. Ballance WC, Qin EC, Chung HJ, Gillette MU, Kong H. Reactive oxygen species-responsive drug delivery systems for the treatment of neurodegenerative diseases. Biomaterials. 2019;217:119292.

23. Zhou Z, Ni K, Deng H, Chen X. Dancing with reactive oxygen species generation and elimination in nanotheranostics for disease treatment. Adv Drug Del Rev. 2020;158:73-90.

24. Bhatt P, Trehan S, Inamdar N, Mourya VK, Misra A. Chapter 1-polymers in drug delivery: an update. In: Misra A, Shahiwala A, editors. Applications of Polymers in Drug Delivery (Second Edition). Elsevier; 2021. p. 1-42.

25. Fang J, Seki T, Maeda H. Therapeutic strategies by modulating oxygen stress in cancer and inflammation. Adv Drug Del Rev. 2009;61(4):290-302.

26. El-Mohtadi F, d'Arcy R, Tirelli N. Oxidation-responsive materials: biological rationale, state of the art, multiple responsiveness, and open issues. Macromol Rapid Commun. 2019;40(1):1800699.

27. Hou J-T, et al. Fluorescent imaging of reactive oxygen and nitrogen species associated with pathophysiological processes. Chem. 2020;6(4):832-66.

28. Broaders KE, Grandhe S, Fréchet JMJ. A biocompatible oxidation-triggered carrier polymer with potential in therapeutics. J Am Chem Soc. 2011;133(4):756-8.

29. de Gracia LC, et al. Biocompatible polymeric nanoparticles degrade and release cargo in response to biologically relevant levels of hydrogen peroxide. J Am Chem Soc. 2012;134(38):15758-64.

30. Zhang D, et al. Biocompatible reactive oxygen species (ROS)responsive nanoparticles as superior drug delivery vehicles. Adv Healthcare Mater. 2015;4(1):69-76.

31. Deng Z, et al. Engineering intracellular delivery nanocarriers and nanoreactors from oxidation-responsive polymersomes via synchronized bilayer cross-linking and permeabilizing inside live cells. J Am Chem Soc. 2016;138(33):10452-66.

32. Tan J, Deng Z, Liu G, Hu J, Liu S. Anti-inflammatory polymersomes of redox-responsive polyprodrug amphiphiles with inflammation-triggered indomethacin release characteristics. Biomaterials. 2018;178:608-19.

33. Feng S, et al. Nanoparticles responsive to the inflammatory microenvironment for targeted treatment of arterial restenosis. Biomaterials. 2016;105:167-84.

34. Zhang Q, et al. A superoxide dismutase/catalase mimetic nanomedicine for targeted therapy of inflammatory bowel disease. Biomaterials. 2016;105:206-21.

35. Dou Y, et al. Non-proinflammatory and responsive nanoplatforms for targeted treatment of atherosclerosis. Biomaterials. 2017;143:93-108.

36. Cheng $\mathrm{J}$, et al. A targeting nanotherapy for abdominal aortic aneurysms. J Am Coll Cardiol. 2018;72(21):2591-605.

37. Wang Y, et al. Targeted therapy of atherosclerosis by a broad-spectrum reactive oxygen species scavenging nanoparticle with intrinsic anti-inflammatory activity. ACS Nano. 2018;12(9):8943-60.

38. Li L, et al. A broad-spectrum ROS-eliminating material for prevention of inflammation and drug-induced organ toxicity. Adv Sci. 2018;5(10):1800781.

39. Hu P, Tirelli N. Scavenging ROS: superoxide dismutase/catalase mimetics by the use of an oxidation-sensitive nanocarrier/ enzyme conjugate. Bioconjugate Chem. 2012;23(3):438-49.

40. Seshadri G, et al. The delivery of superoxide dismutase encapsulated in polyketal microparticles to rat myocardium and protection from myocardial ischemia-reperfusion injury. Biomaterials. 2010;31(6):1372-9.

41. Ni R, et al. Reactive oxygen species-responsive dexamethasoneloaded nanoparticles for targeted treatment of rheumatoid arthritis via suppressing the $i$ Rhom $2 / \mathrm{TNF}-\alpha / \mathrm{BAFF}$ signaling pathway. Biomaterials. 2020;232:119730.

42. Hu B, Dai F, Fan Z, Ma G, Tang Q, Zhang X. Nanotheranostics: Congo Red/Rutin-MNPs with enhanced magnetic resonance imaging and H2O2-responsive therapy of Alzheimer's disease in APPswe/PS1dE9 transgenic mice. Adv Mater. 2015;27(37):5499-505.

43. Lu Y, et al. Microenvironment remodeling micelles for Alzheimer's disease therapy by early modulation of activated microglia. Adv Sci. 2019;6(4):1801586.

44. Bush AI. The metallobiology of Alzheimer's disease. Trends Neurosci. 2003;26(4):207-14.

45. Klafki H-W, Staufenbiel M, Kornhuber J, Wiltfang J. Therapeutic approaches to Alzheimer's disease. Brain. 2006;129(11):2840-55.

46. Liu G, Garrett MR, Men P, Zhu X, Perry G, Smith MA. Nanoparticle and other metal chelation therapeutics in Alzheimer disease. Biochimica et Biophysica Acta (BBA) - Molecular Basis of Disease. 2005;1741(3):246-52.

47. Geng J, Li M, Wu L, Chen C, Qu X. Mesoporous silica nanoparticle-based $\mathrm{H} 2 \mathrm{O} 2$ responsive controlled-release system used for Alzheimer's disease treatment. Adv Healthcare Mater. 2012;1(3):332-6.

48. Li M, Shi P, Xu C, Ren J, Qu X. Cerium oxide caged metal chelator: anti-aggregation and anti-oxidation integrated $\mathrm{H} 2 \mathrm{O} 2$ responsive controlled drug release for potential Alzheimer's disease treatment. Chem Sci. 2013;4(6):2536-42.

49. Gao C, et al. Treatment of atherosclerosis by macrophagebiomimetic nanoparticles via targeted pharmacotherapy and sequestration of proinflammatory cytokines. Nat Commun. 2020;11(1):2622.

50. Wilson DS, Dalmasso G, Wang L, Sitaraman SV, Merlin D, Murthy N. Orally delivered thioketal nanoparticles loaded with TNF- $\alpha$-siRNA target inflammation and inhibit gene expression in the intestines. Nat Mater. 2010;9(11):923-8.

51. Napoli A, Valentini M, Tirelli N, Muller M, Hubbell JA. Oxidationresponsive polymeric vesicles. Nat Mater. 2004;3(3):183-9. 
52. Rehor A, Hubbell JA, Tirelli N. Oxidation-sensitive polymeric nanoparticles. Langmuir. 2005;21(1):411-7.

53. Napoli A, Tirelli N, Wehrli E, Hubbell JA. Lyotropic behavior in water of amphiphilic ABA triblock copolymers based on poly(propylene sulfide) and poly(ethylene glycol). Langmuir. 2002;18(22):8324-9.

54. Gupta MK, Meyer TA, Nelson CE, Duvall CL. Poly(PS-b-DMA) micelles for reactive oxygen species triggered drug release. $\mathrm{J}$ Controlled Release. 2012;162(3):591-8.

55. Wu T, et al. Aortic plaque-targeted andrographolide delivery with oxidation-sensitive micelle effectively treats atherosclerosis via simultaneous ROS capture and anti-inflammation. Nanomed Nanotechnol Biol Med. 2018;14(7):2215-26.

56. An L, et al. Inflammation-targeted celastrol nanodrug attenuates collagen-induced arthritis through NF-kappaB and Notch1 pathways. Nano Lett. 2020;20(10):7728-36.

57. Yi S, et al. Tailoring nanostructure morphology for enhanced targeting of dendritic cells in atherosclerosis. ACS Nano. 2016;10(12):11290-303

58. Allen SD, et al. Celastrol-loaded PEG-b-PPS nanocarriers as an anti-inflammatory treatment for atherosclerosis. Biomater Sci. 2019;7(2):657-68.

59. Yi S, et al. Surface engineered polymersomes for enhanced modulation of dendritic cells during cardiovascular immunotherapy. Adv Funct Mater. 2019;29(42):1904399.

60. Yi S, et al. An injectable hydrogel platform for sustained delivery of anti-inflammatory nanocarriers and induction of regulatory $\mathrm{T}$ cells in atherosclerosis. Front Bioeng Biotech. 2020;8:542.

61. Ma N, Li Y, Xu H, Wang Z, Zhang X. Dual redox responsive assemblies formed from diselenide block copolymers. J Am Chem Soc. 2010;132(2):442-3.

62. Sun C, Tan Y, Xu H. From selenite to diselenide-containing drug delivery systems. ACS Mater Lett. 2020;2(9):1173-7.

63. Yang L, Sun J, Xie W, Liu Y, Liu J. Dual-functional selenium nanoparticles bind to and inhibit amyloid beta fiber formation in Alzheimer's disease. J Mater Chem B. 2017;5(30):5954-67.

64. Li Y, et al. Positively charged polyprodrug amphiphiles with enhanced drug loading and reactive oxygen species-responsive release ability for traceable synergistic therapy. J Am Chem Soc. 2018;140(11):4164-71.

65. Lee $\mathrm{D}$, et al. In vivo imaging of hydrogen peroxide with chemiluminescent nanoparticles. Nat Mater. 2007;6(10):765-9.

66. Shuhendler AJ, Pu K, Cui L, Uetrecht JP, Rao J. Real-time imaging of oxidative and nitrosative stress in the liver of live animals for drug-toxicity testing. Nat Biotechnol. 2014;32(4):373-80.

67. Yang J, et al. Oxalate-curcumin-based probe for micro- and macroimaging of reactive oxygen species in Alzheimer's disease. Proc Natl Acad Sci U S A. 2017;114(47):12384-9.

68. Park $\mathrm{H}$, et al. Antioxidant and anti-inflammatory activities of hydroxybenzyl alcohol releasing biodegradable polyoxalate nanoparticles. Biomacromol. 2010;11(8):2103-8.

69. Kim S, et al. Reduction of oxidative stress by p-hydroxybenzyl alcohol-containing biodegradable polyoxalate nanoparticulate antioxidant. Biomaterials. 2011;32(11):3021-9.

70. Yoo D, Guk K, Kim H, Khang G, Wu D, Lee D. Antioxidant polymeric nanoparticles as novel therapeutics for airway inflammatory diseases. Int J Pharm. 2013;450(1-2):87-94.

71. Mei J, Leung NLC, Kwok RTK, Lam JWY, Tang BZ. Aggregationinduced emission: together we shine, united we soar! Chem Rev. 2015;115(21):11718-940.

72. Ma B, Xu H, Zhuang W, Wang Y, Li G, Wang Y. Reactive oxygen species responsive theranostic nanoplatform for two-photon aggregation-induced emission imaging and therapy of acute and chronic inflammation. ACS Nano. 2020;14(5):5862-73.

73. Chung M-F, Chia W-T, Wan W-L, Lin Y-J, Sung H-W. Controlled release of an anti-inflammatory drug using an ultrasensitive
ROS-responsive gas-generating carrier for localized inflammation inhibition. J Am Chem Soc. 2015;137(39):12462-5.

74. Lee Y, Kim H, Kang S, Lee J, Park J, Jon S. Bilirubin nanoparticles as a nanomedicine for anti-inflammation therapy. Angew Chem, Int Ed. 2016;55(26):7460-3.

75. Kim DE, Lee Y, Kim M, Lee S, Jon S, Lee S-H. Bilirubin nanoparticles ameliorate allergic lung inflammation in a mouse model of asthma. Biomaterials. 2017;140:37-44.

76. Keum $\mathrm{H}$, et al. Bilirubin nanomedicine alleviates psoriatic skin inflammation by reducing oxidative stress and suppressing pathogenic signaling. J Controlled Release. 2020;325:359-69.

77. $\mathrm{Wu} \mathrm{G}$, et al. Molecularly engineered macrophage-derived exosomes with inflammation tropism and intrinsic heme biosynthesis for atherosclerosis treatment. Angew Chem, Int Ed. 2020;59(10):4068-74.

78. Guo J, et al. Cyclodextrin-derived intrinsically bioactive nanoparticles for treatment of acute and chronic inflammatory diseases. Adv Mater. 2019;31(46):e1904607.

79. Garlick PB, Radda GK, Seeley PJ. Studies of acidosis in the ischaemic heart by phosphorus nuclear magnetic resonance. Biochem J. 1979;184(3):547-54.

80. Walters G, Pountos I, Giannoudis PV. The cytokines and microenvironment of fracture haematoma: Current evidence. J Tissue Eng Regen Med. 2018;12(3):e1662-77.

81. Gao W, Chan JM, Farokhzad OC. pH-responsive nanoparticles for drug delivery. Mol Pharmaceutics. 2010;7(6):1913-20.

82. Casey JR, Grinstein S, Orlowski J. Sensors and regulators of intracellular pH. Nat Rev Mol Cell Biol. 2010;11(1):50-61.

83. Heffernan MJ, Murthy N. Polyketal nanoparticles: a new pHsensitive biodegradable drug delivery vehicle. Bioconjugate Chem. 2005;16(6):1340-2.

84. Lee S, Yang SC, Heffernan MJ, Taylor WR, Murthy N. Polyketal microparticles: a new delivery vehicle for superoxide dismutase. Bioconjugate Chem. 2007;18(1):4-7.

85. Sy JC, et al. Sustained release of a 338 inhibitor from non-inflammatory microspheres inhibits cardiac dysfunction. Nat Mater. 2008;7(11):863-8.

86. Somasuntharam I, et al. Delivery of Nox2-NADPH oxidase siRNA with polyketal nanoparticles for improving cardiac function following myocardial infarction. Biomaterials. 2013;34(31):7790-8.

87. Gillies ER, Fréchet JMJ. A new approach towards acid sensitive copolymer micelles for drug delivery. Chem Commun. 2003; $14: 1640-1$.

88. Gillies ER, Jonsson TB, Fréchet JMJ. Stimuli-responsive supramolecular assemblies of linear-dendritic copolymers. J Am Chem Soc. 2004; 126(38):11936-43.

89. Bachelder EM, Beaudette TT, Broaders KE, Dashe J, Frechet JM. Acetal-derivatized dextran: an acid-responsive biodegradable material for therapeutic applications. J Am Chem Soc. 2008;130(32):10494-5.

90. Griset AP, Walpole J, Liu R, Gaffey A, Colson YL, Grinstaff MW. Expansile nanoparticles: synthesis, characterization, and in vivo efficacy of an acid-responsive polymeric drug delivery system. J Am Chem Soc. 2009;131(7):2469-71.

91. Zhang J, Jia Y, Li X, Hu Y, Li X. Facile engineering of biocompatible materials with $\mathrm{pH}$-modulated degradability. Adv Mater. 2011;23(27):3035-40.

92. Liu X-M, et al. Synthesis and evaluation of a well-defined HPMA copolymer-dexamethasone conjugate for effective treatment of rheumatoid arthritis. Pharm Res. 2008;25(12):2910-9.

93. Liu X-M, Quan L-d, Tian J, Laquer FC, Ciborowski P, Wang D. Syntheses of click PEG-dexamethasone conjugates for the treatment of rheumatoid arthritis. Biomacromolecules. 2010;11(10):2621-8. 
94. Quan L, et al. Nanomedicines for inflammatory arthritis: headto-head comparison of glucocorticoid-containing polymers, micelles, and liposomes. ACS Nano. 2013;8(1):458-66.

95. Ren K, Yuan H, Zhang Y, Wei X, Wang D. Macromolecular glucocorticoid prodrug improves the treatment of dextran sulfate sodium-induced mice ulcerative colitis. Clin Immunol. 2015;160(1):71-81.

96. Crielaard BJ, et al. Glucocorticoid-loaded core-cross-linked polymeric micelles with tailorable release kinetics for targeted therapy of rheumatoid arthritis. Angew Chem, Int Ed. 2012;51(29):7254-8.

97. Li C, et al. pH-sensitive polymeric micelles for targeted delivery to inflamed joints. J Controlled Release. 2017;246:133-41.

98. Turecek PL, Bossard MJ, Schoetens F, Ivens IA. PEGylation of biopharmaceuticals: a review of chemistry and nonclinical safety information of approved drugs. J Pharm Sci. 2016;105(2):460-75.

99. Kong L, Campbell F, Kros A. DePEGylation strategies to increase cancer nanomedicine efficacy. Nanoscale Horizons. 2019;4(2):378-87.

100. Aldayel AM, et al. Acid-sensitive sheddable PEGylated PLGA nanoparticles increase the delivery of TNF- $\alpha$ siRNA in chronic inflammation sites. Molecular Therapy-Nucleic Acids. 2016;5:e340.

101. O'Mary HL, et al. Acid-sensitive sheddable PEGylated, mannosemodified nanoparticles increase the delivery of betamethasone to chronic inflammation sites in a mouse model. Mol Pharmaceutics. 2017;14(6):1929-37.

102. Brown KE, Shao W, Bathon J, Leong KW. Controlled drug delivery to the joints by enzymatically degradable microspheres. MRS Proc. 1993;331:73.

103. Brown KE, et al. Gelatin/chondroitin 6-sulfate microspheres for the delivery of therapeutic proteins to the joint. Arthritis Rheum. 1998;41(12):2185-95.

104. Park E, Hart ML, Rolauffs B, Stegemann JP, R TA. Bioresponsive microspheres for on-demand delivery of anti-inflammatory cytokines for articular cartilage repair. J Biomed Mater Res Part A. 2020;108(3):722-33.

105. Li Y, Rodrigues J, Tomás H. Injectable and biodegradable hydrogels: gelation, biodegradation and biomedical applications. Chem Soc Rev. 2012;41(6):2193-221.

106. Aimetti AA, Tibbitt MW, Anseth KS. Human neutrophil elastase responsive delivery from poly(ethylene glycol) hydrogels. Biomacromol. 2009;10(6):1484-9.

107. Mejias JC et al. Neutrophil-targeted, protease-activated pulmonary drug delivery blocks airway and systemic inflammation. JCI Insight. 2019;4(23).

108. Du X, Zhou J, Shi J, Xu B. Supramolecular hydrogelators and hydrogels: from soft matter to molecular biomaterials. Chem Rev. 2015;115(24):13165-307.

109. Li X, Li J, Gao Y, Kuang Y, Shi J, Xu B. Molecular nanofibers of olsalazine form supramolecular hydrogels for reductive release of an anti-inflammatory agent. J Am Chem Soc. 2010;132(50):17707-9.

110. Vemula PK, et al. On-demand drug delivery from self-assembled nanofibrous gels: a new approach for treatment of proteolytic disease. J Biomed Mater Res Part A. 2011;97A(2):103-10.

111. Gajanayake $\mathrm{T}$ et al. A single localized dose of enzyme-responsive hydrogel improves long-term survival of a vascularized composite allograft. Sci Transl Med. 2014;6(249):249ra110.

112. Zhang $\mathrm{S}$ et al. An inflammation-targeting hydrogel for local drug delivery in inflammatory bowel disease. Sci Transl Med. 2015;7(300):300ra128.

113. Joshi N, et al. Towards an arthritis flare-responsive drug delivery system. Nat Commun. 2018;9(1):1275.
114. Homma A, et al. Novel hyaluronic acid-methotrexate conjugates for osteoarthritis treatment. Biorg Med Chem. 2009;17(13):4647-56.

115. Homma A, et al. Synthesis and optimization of hyaluronic acidmethotrexate conjugates to maximize benefit in the treatment of osteoarthritis. Biorg Med Chem. 2010;18(3):1062-75.

116. Wei J, et al. Design, synthesis and biological evaluation of enzymatically cleavable NSAIDs prodrugs derived from selfimmolative dendritic scaffolds for the treatment of inflammatory diseases. Biorg Med Chem. 2013;21(14):4192-200.

117. Ye $\mathrm{J}$ et al. An enzyme-responsive prodrug with inflammationtriggered therapeutic drug release characteristics. Macromol Biosci. 2020:e2000116.

118. Deng Z, Hu J, Liu S. Disulfide-based self-immolative linkers and functional bioconjugates for biological applications. Macromol Rapid Commun. 2020;41(1):1900531.

119. Xiao B, et al. Mannosylated bioreducible nanoparticle-mediated macrophage-specific TNF- $\alpha$ RNA interference for IBD therapy. Biomaterials. 2013;34(30):7471-82.

120. $\mathrm{Xu} \mathrm{X}$, et al. Efficient and targeted drug/siRNA co-delivery mediated by reversibly crosslinked polymersomes toward anti-inflammatory treatment of ulcerative colitis (UC). Nano Res. 2019;12(3):659-67.

121. Poh S, Lin JB, Panitch A. Release of anti-inflammatory peptides from thermosensitive nanoparticles with degradable cross-links suppresses pro-inflammatory cytokine production. Biomacromol. 2015;16(4):1191-200.

122. Feng N, Yang M, Feng X, Wang Y, Chang F, Ding J. Reductionresponsive polypeptide nanogel for intracellular drug delivery in relieving collagen-induced arthritis. ACS Biomater Sci Eng. 2018;4(12):4154-62.

123. Yin $\mathrm{N}$, et al. Intra-articular injection of indomethacin-methotrexate in situ hydrogel for the synergistic treatment of rheumatoid arthritis. J Mater Chem B. 2020;8(5):993-1007.

124. Zhuang J, Gordon MR, Ventura J, Li L, Thayumanavan S. Multistimuli responsive macromolecules and their assemblies. Chem Soc Rev. 2013;42(17).

125. Schattling $P$, Jochum FD, Theato P. Multi-stimuli responsive polymers - the all-in-one talents. Polym Chem. 2014;5(1):25-36.

126. Fu X, Hosta-Rigau L, Chandrawati R, Cui J. Multi-stimuliresponsive polymer particles, films, and hydrogels for drug delivery. Chem. 2018;4(9):2084-107.

127. Mahmoud EA, Sankaranarayanan J, Morachis JM, Kim G, Almutairi A. Inflammation responsive logic gate nanoparticles for the delivery of proteins. Bioconjugate Chem. 2011;22(7):1416-21.

128. Lin L, et al. Nanodrug with ROS and $\mathrm{pH}$ dual-sensitivity ameliorates liver fibrosis via multicellular regulation. Adv Sci. 2020;7(7):1903138.

129. Kwon J, Kim J, Park S, Khang G, Kang PM, Lee D. Inflammationresponsive antioxidant nanoparticles based on a polymeric prodrug of vanillin. Biomacromol. 2013;14(5):1618-26.

130. Daniel KB, Callmann CE, Gianneschi NC, Cohen SM. Dualresponsive nanoparticles release cargo upon exposure to matrix metalloproteinase and reactive oxygen species. Chem Commun. 2016;52(10):2126-8.

131. Callmann CE, Barback CV, Thompson MP, Hall DJ, Mattrey RF, Gianneschi NC. Therapeutic enzyme-responsive nanoparticles for targeted delivery and accumulation in tumors. Adv Mater. 2015;27(31):4611-5.

132. Nguyen MM, et al. Enzyme-responsive nanoparticles for targeted accumulation and prolonged retention in heart tissue after myocardial infarction. Adv Mater. 2015;27(37):5547-52.

133. $\mathrm{Pu} \mathrm{HL}$, et al. Nanoparticles with dual responses to oxidative stress and reduced ph for drug release and anti-inflammatory applications. ACS Nano. 2014;8(2):1213-21. 
134. Lee S, Stubelius A, Hamelmann N, Tran V, Almutairi A. Inflammation-responsive drug-conjugated dextran nanoparticles enhance anti-inflammatory drug efficacy. ACS Appl Mater Interfaces. 2018;10(47):40378-87.

135. Zhang $\mathrm{R}$, et al. A $\mathrm{pH} / \mathrm{ROS}$ dual-responsive and targeting nanotherapy for vascular inflammatory diseases. Biomaterials. 2020;230:119605.

136. Bertoni $\mathrm{S}$, et al. $\mathrm{pH}$ and reactive oxygen species-sequential responsive nano-in-micro composite for targeted therapy of inflammatory bowel disease. Adv Funct Mater. 2018;28(50):1806175.
137. Anselmo AC, Mitragotri S. Nanoparticles in the clinic: an update. 2019;4(3):e10143.

Publisher's Note Springer Nature remains neutral with regard to jurisdictional claims in published maps and institutional affiliations. 